\title{
Reciprocal regulation of STING and TCR signaling by mTORC1 for T-cell activation and function
}

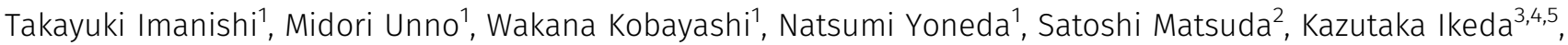 \\ Takayuki Hoshii ${ }^{6}$, Atsushi Hirao ${ }^{6}$, Kensuke Miyake', Glen N Barber ${ }^{8}$, Makoto Arita ${ }^{3,5,9}$, Ken J Ishii ${ }^{10,11}$, Shizuo Akira ${ }^{12}$, \\ Takashi Saito ${ }^{1,13}$ (1)
}

\begin{abstract}
Stimulator of interferon genes (STING) plays a key role in detecting cytosolic DNA and induces type I interferon (IFN-I) responses for host defense against pathogens. Although T cells highly express STING, its physiological role remains unknown. Here, we show that costimulation of T cells with the STING ligand CGAMP and TCR leads to IFN-I production and strongly inhibits T-cell growth. TCRmediated $\mathrm{mTORC} 1$ activation and sustained activation of IRF3 are required for CGAMP-induced IFN-I production, and the mTORC1 activity is partially counteracted by CGAMP, thereby blocking proliferation. This mTORC1 inhibition in response to costimulation depends on IRF 3 and IRF7. Effector T cells produce much higher IFN-I levels than innate cells in response to cGAMP. Finally, we demonstrated that STING stimulation in T cells is effective in inducing antitumor responses in vivo. Our studies demonstrate that the outputs of STING and TCR signaling pathways are mutually regulated through mTORC1 to modulate T-cell functions.
\end{abstract}

DOI 10.26508/lsa.201800282 | Received 17 December 2018 | Revised 26 December 2018 | Accepted 7 January 2019 | Published online 25 January 2019

\section{Introduction}

In addition to the antigen-specific TCR signals, T-cell activation is regulated by several different signals through costimulatory receptors. The most critical positive costimulatory signal is mediated by CD28 upon interaction with its ligands CD80/86 on APCs. By contrast, the inhibitory costimulatory receptors, cytotoxic T lymphocyte- associated protein 4 (CTLA-4) and programmed cell death 1 (PD-1) deliver negative signals to terminate T-cell responses and prevent autoimmune responses. The balance between these positive and negative costimulation signals determines the activation state, differentiation, and functions of T cells (Chen \& Flies, 2013).

Mechanistic target of rapamycin (mTOR) is activated by TCR/ CD28 signals and environmental signals and regulates cellular metabolism and protein synthesis through downstream pathways, such as $4 \mathrm{E}-\mathrm{BP} 1$ and $\mathrm{S} 6$ kinase, and integrates these signals to regulate T-cell proliferation and differentiation (Chi, 2012). mTOR interacts with several proteins to form mTOR complex-1 (mTORC1) and -2 (mTORC2), which contain the essential scaffold protein Raptor and Rictor, respectively. T cell-specific gene deletion analyses revealed that mTORC1 has a central role for T-cell activation, differentiation, and antigen-specific immune responses in vivo (Yang et al, 2013).

Toll-like receptors (TLRs) are primary sensors in the innate immune system and recognize pathogen-associated molecular patterns (Takeda et al, 2003) to produce inflammatory cytokines and induce up-regulation of MHC and costimulatory molecules on APCS (Medzhitov, 2001). TLRs are also expressed by T cells, where they can have costimulatory functions. Indeed, TLR2 ligands enhance T-cell proliferation upon TCR stimulation (Komai-Koma et al, 2004; Cottalorda et al, 2006), directly trigger Th1 effector functions without TCR stimulation (Imanishi et al, 2007), and promote Th17 responses (Reynolds et al, 2010). Furthermore, we have shown that nucleic acids induce costimulation signals for Th2 differentiation independently of any known nucleic acid sensors, including TLRS, RIG-I-like receptors (RLRS), inflammasomes, and STING (Imanishi et al, 2014).

\footnotetext{
'Laboratory for Cell Signaling, RIKEN Center for Integrative Medical Sciences, Yokohama, Japan ${ }^{2}$ Department of Cell Signaling, Institute of Biomedical Sciences, Kansai Medical University, Hirakata, Japan ${ }^{3}$ Laboratory for Metabolomics, RIKEN Center for Integrative Medical Sciences, Yokohama, Japan ${ }^{4} J a p a n$ Agency for Medical Research and Development (AMED)-PRIME, Japan Agency for Medical Research and Development, Tokyo, Japan ${ }^{5}$ Graduate School of Medical Life Science, Yokohama City University, Yokohama, Japan ${ }^{6}$ Division of Molecular Genetics, Cancer Research Institute, Kanazawa University, Kanazawa, Japan ${ }^{7}$ Division of Innate Immunity, Department of Microbiology and Immunology, Institute of Medical Science, University of Tokyo, Tokyo, Japan ${ }^{8}$ Department of Cell Biology and the Sylvester Comprehensive Cancer Center, University of Miami Miller School of Medicine, Miami, FL, USA ${ }^{9}$ Division of Physiological Chemistry and Metabolism, Graduate School of Pharmaceutical Sciences, Keio University, Tokyo, Japan ${ }^{10}$ Laboratory of Vaccine Science, World Premier International Research Center Initiative (WPI) Immunology Frontier Research Center, Osaka University, Suita, Japan ${ }^{11}$ Laboratory of Adjuvant Innovation, National Institutes of Biomedical Innovation, Health and Nutrition, Osaka, Japan ${ }^{12}$ Laboratory of Host Defense, WPI Immunology Frontier Research Center, Osaka University, Osaka, Japan ${ }^{13}$ Laboratory for Cell Signaling, WPI Immunology Frontier Research Center, Osaka University, Osaka, Japan
}

Correspondence: takashi.saito@riken.jp; takayuki.imanishi@riken.jp

Wakana Kobayashi's present address is Laboratory for Mucosal Immunity, RIKEN Center for Integrative Medical Sciences, Yokohama, Japan.

Takayuki Hoshii's present address is Department of Pediatric Oncology, Dana-Farber Cancer Institute and Division of Hematology/Oncology, Boston Children's Hospital, Harvard Medical School, Boston, USA and Center for Epigenetics Research, Memorial Sloan Kettering Cancer Center, New York, USA 
STING is a pattern recognition receptor localized in the ER membrane (Ishikawa \& Barber, 2008) and recognizes cyclic dinucleotides (CDNs) derived from bacteria, resulting in induction of IFN-I responses (Burdette et al, 2011). STING also plays a central role in detecting cytosolic viral DNA (Ishikawa \& Barber, 2008; Ishikawa et al, 2009). DNA derived from pathogens and even self-DNA (Gao et al, 2015) are recognized by the cyclic GMP-AMP (cGAMP) synthase (cGAS) (Sun et al, 2013), which catalyzes the conversion of GTP and ATP into the second messenger 2'3' cGAMP (Wu et al, 2013), which binds to and activates STING.

In this study, we assessed the function of STING in T cells and demonstrated that STING activation induces suppression of T-cell proliferation through inhibiting TCR-induced mTORC1 activation. STING-mediated inhibition of mTORC1 is dependent on IRF3/7 but not TBK1/IKKE. We also found that naive T cells produce IFN-I upon STING and TCR stimulation. Mechanistically, TCR stimulation induces the sustained activation of IRF3 and provides the signals for mTORC1 activation for IFN-I responses. Our data show the central role of MTORC1 in STING-mediated proliferation inhibition and IFN-I responses in T cells. Finally, we demonstrated that STING in T cells is crucial for antitumor immune responses.

\section{Results}

\section{Activation of STING in T cells inhibits growth}

Naive $\mathrm{CD}^{+} \mathrm{T}$ cells express STING protein at levels similar to BMDCS (Fig S1A), suggesting their intrinsic function in $T$ cells as pattern recognition receptors. Whereas TLR ligands directly enhance T-cell proliferation upon TCR stimulation (Komai-Koma et al, 2004; Cottalorda et al, 2006), we found that STING ligands such as CGAMP and DMXAA strongly inhibit proliferation of naive $C D 4^{+} \mathrm{T}$ cells upon stimulation with anti-CD3/CD28 (Fig 1A). Studies with STINGdeficient (KO) mice confirmed that this suppression is STING dependent. Similar results were obtained with naive $\mathrm{CD} 8^{+} \mathrm{T}$ cells (Fig S1B). Notably, CGAMP inhibited T-cell proliferation without lipofection similarly to DMXAA, which has a cell-permeable structure. The inhibition of proliferation by cGAMP was also observed in an antigen-specific system, using T cells from Ovalbumin (OVA)specific OT-II Tg mice (Fig S1C). Although proliferation was inhibited, the percentage of live cells in these cultures did not change in the presence of STING ligands except for those stimulated with high doses of DMXAA (Fig 1B). Consistently, only high concentrations of

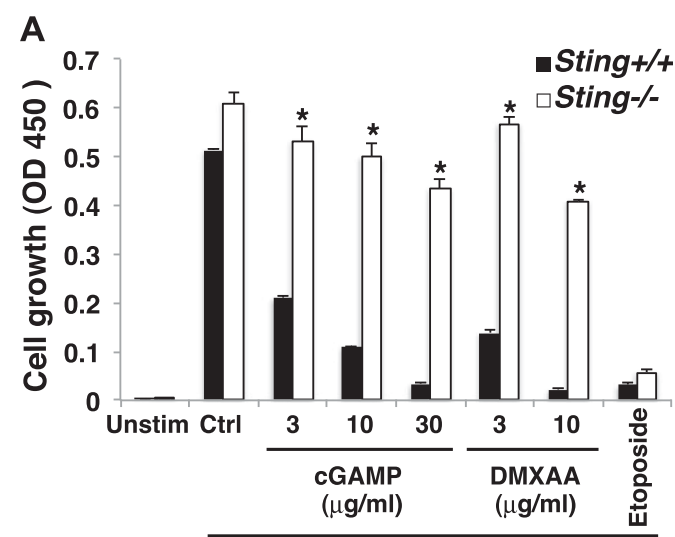

D

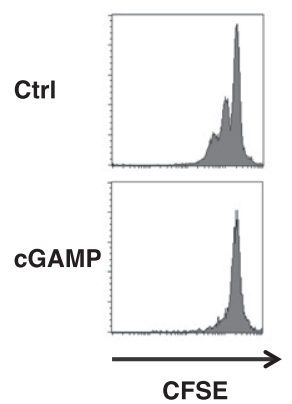

Anti-CD3/28

$\mathrm{E}$

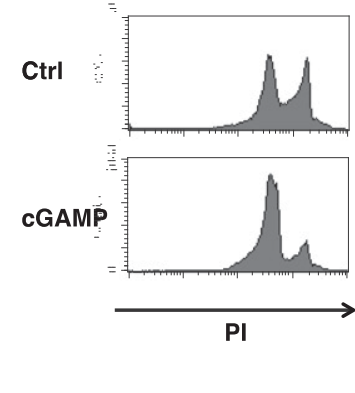

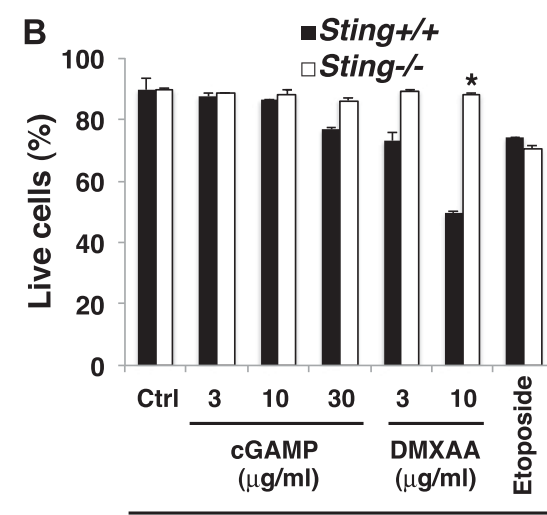

Anti-CD3/28

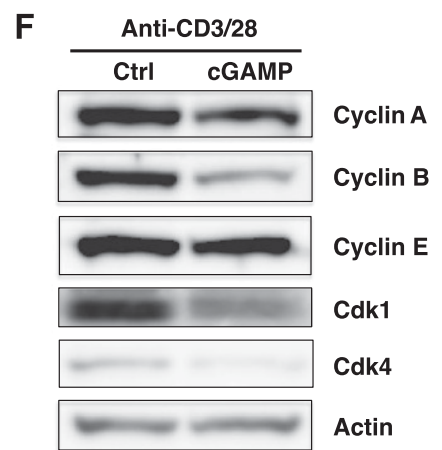

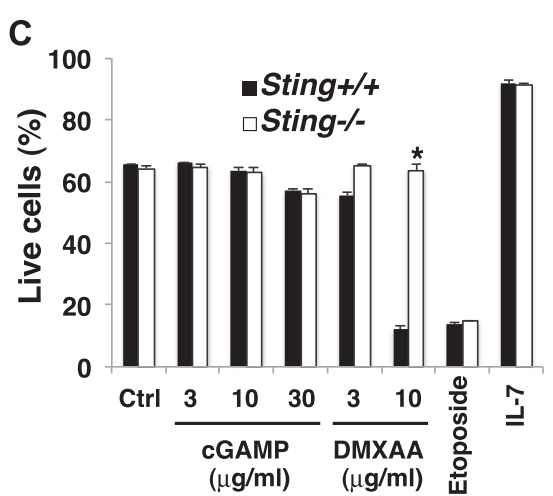

Figure 1. STING stimulation in T cells inhibits cell cycle progression.

(A, B) FACS-sorted naive $\mathrm{CD}^{+} \mathrm{T}$ cells from Sting ${ }^{+/+}$or Sting $^{-/-}$mice were stimulated with immobilized anti-CD3E plus anti-CD28 (anti-CD3/CD28) Abs with or without STING ligands CGAMP or DMXAA. Cell growth was assessed after $48 \mathrm{~h}$ of stimulation by a WST-8 proliferation assay (A), and cell survival was determined after $18 \mathrm{~h}$ of stimulation by Propidium iodide (PI) and Annexin V staining (B). (C) Naive CD $4^{+}$T cells from Sting ${ }^{+/+}$or Sting $^{-/-}$mice were stimulated with cGAMP or DMXAA for $18 \mathrm{~h}$, and cell survival was determined by PI and Annexin V staining. (D, E) Naive CD4 ${ }^{+}$T cells were stimulated with anti-CD3/CD28 Abs with or without cGAMP for 48 h, and cell division was determined by a CFSE-labeling assay (D) and cell cycle was assessed by PI staining (E). (F) Western blot analysis for protein expression of cell cycle-related genes in $\mathrm{CD}^{+} \mathrm{T}$ cells upon stimulation with anti-CD3/CD28 Abs with or without CGAMP for $24 \mathrm{~h}$. Data are the mean from duplicate (A-C) \pm SD. Data are representative of at least three independent experiments. (A-F) ${ }^{\star} P<0.05, t$ test (compared with WT cells treated with indicated ligands). 
DMXAA induced cell death as efficiently as etoposide in the absence of TCR stimulation (Fig 1C). We also found that treatment with a pancaspase inhibitor Z-VAD or use of RIP3 (critical kinase responsible for necroptosis)-KO T cells did not affect the CGAMP-induced growth inhibition upon TCR stimulation (Fig S1D and E). These data suggest that CGAMP as the natural ligand for STING may inhibit proliferation of T cells through growth arrest rather than cell death. To test this possibility, naive $\mathrm{CD}^{+} \mathrm{T}$ cells were labeled with 5-(and -6)Carboxyfluorescein diacetate succinimidyl ester (CFSE) and stimulated with anti-CD3/CD28 with or without CGAMP. Cell division was severely impaired by CGAMP (Fig 1D), and activated $\mathrm{CD}^{+} \mathrm{T}$ cells remained in the G0-G1 phase of the cell cycle in the presence of CGAMP (Fig 1E), indicating that costimulation of T cells with TCR and cGAMP induced cell cycle arrest. Consistently, mRNA and protein expression of cell cycle-related genes such as cyclins A2, B1, D2, Cdk1, and Cdk4 were reduced in the presence of CGAMP in STINGdependent fashion (Fig S1F and 1F). Conversely, the expression of CDK inhibitor p21 (Cdkn1a) and p27 Kip1 (Cdkn1b) was up-regulated by CGAMP (Fig S1F and G). These data indicate that STING activation in T cells induces cell cycle arrest by modulating the expression of cell cycle-related genes.

\section{STING signals inhibit activation of mTORC1}

Because mTORC1 signaling is required for cell cycle regulation in $T$ cells through the induction of cell cycle-related genes (Yang et al, 2013), it seemed possible that the cell cycle arrest by cGAMP was mediated by the inhibition of mTORC1 activation upon TCR/CD28 stimulation. Indeed, CGAMP strongly inhibited the activation of mTORC1 downstream signaling molecules such as S6K1 and 4E-BP1 upon anti-CD3/28 stimulation (Fig 2A). By contrast, Akt activation was modestly enhanced (Fig 2A), which is similarly observed in Raptor-KO T cells (Yang et al, 2013). Interestingly, IL-2R signaling events, including phosphorylation of STAT5 and JAK3, were also inhibited (Fig 2A). We used STING-KO T cells to confirm that this CGAMP-induced inhibition of both mTORC1 and IL-2R signaling was
A

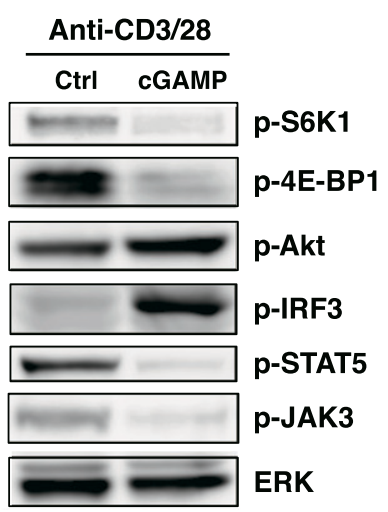

B

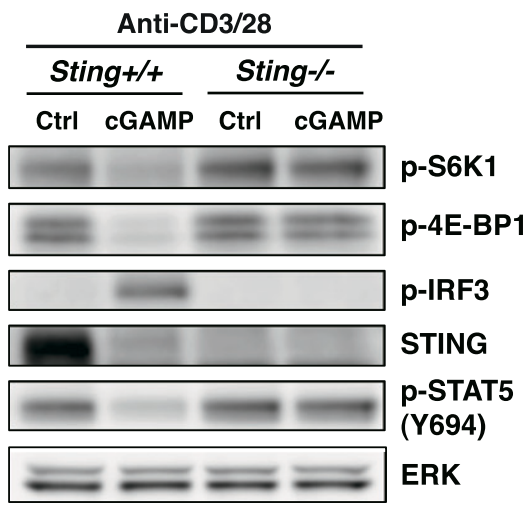

C

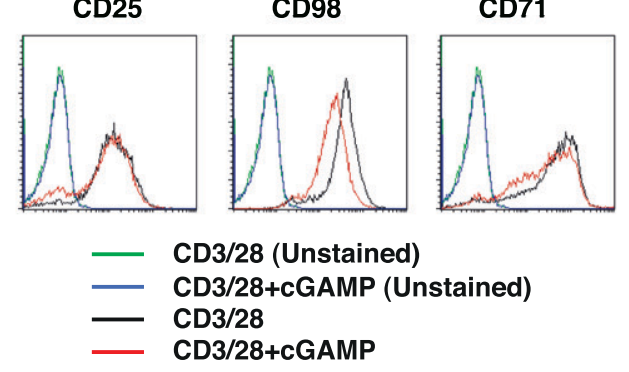

E



D

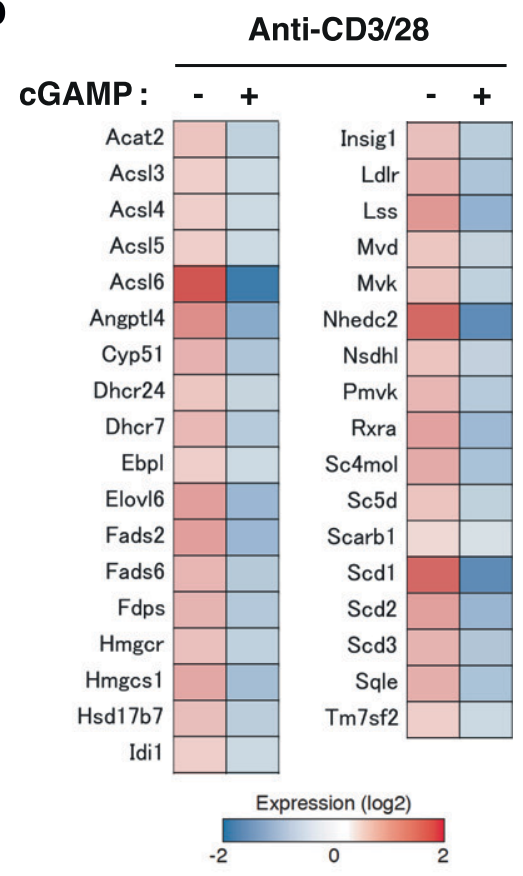

$\overline{A n t i-C D 3 / 28}$

Figure 2. STING activation leads to the inhibition of the mTORC1 pathway.

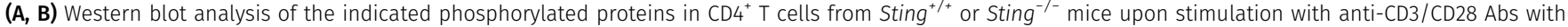
or without cGAMP for $24 \mathrm{~h}$. (C) FACS analysis of indicated surface molecules on CD4 ${ }^{+} \mathrm{T}$ cells upon stimulation with anti-CD3/CD28 Abs with or without cGAMP for $24 \mathrm{~h}$. (D) RNA-seq data of the expression of lipid synthesis-related genes in CD4 $4^{+} \mathrm{T}$ cells upon stimulation with anti-CD3/CD28 Abs with or without cGAMP for 24 h. (E) Quantification of total cholesterol ester (upper) and total DAG (lower) in CD4 ${ }^{+} \mathrm{T}$ cells upon stimulation with anti-CD3/CD28 Abs with or without cGAMP for $24 \mathrm{~h}$, assessed

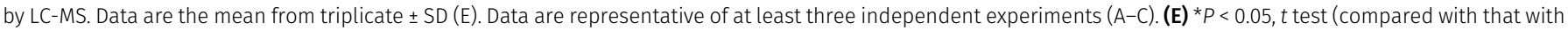
anti-CD3/28 alone). 
dependent on STING (Fig 2B). Although TCR-induced IL-2 production was slightly reduced by CGAMP upon TCR stimulation (Fig S2A), the addition of exogenous IL-2 failed to restore the inhibition of either cGAMP-induced cell growth or STAT5 activation (Fig S2B), suggesting that STING stimulation inhibits the activation of STAT5 independently of IL-2 production. Anti-IL-2R blocking Ab inhibited the phosphorylation of STAT5 but not 4E-BP1 (Fig S2C), indicating that STING activation by CGAMP inhibits TCR-induced mTORC1 signaling and IL-2-induced STAT5 activation. Notably, anti-IL-2R blocking Ab did not inhibit T-cell proliferation upon anti-CD3/28 stimulation in our experimental system, suggesting that cGAMP-induced inhibition of STAT5 activation was not required for STING-mediated growth inhibition (Fig S2D). We confirmed that other STING ligands, cyclic di-AMP and DMXAA, also inhibited both mTORC1 and IL-2R signaling (Fig S2E). mTORC1 regulates the expression of the amino acid transporter CD98 and the transferrin receptor CD71 (Yang et al, 2013), and we found that TCR-induced expression of both CD98 and CD71, but not CD25, was impaired by CGAMP (Fig 2C). These data indicate that CGAMP specifically inhibits both mTORC1 and IL-2 pathways upon TCR stimulation.

Pathway analysis of genes with down-regulation in $\mathrm{CD}^{+} \mathrm{T}$ cells stimulated with anti-CD3/28 plus CGAMP as compared to those stimulated with anti-CD3/28 alone showed that CGAMP stimulation reduced the expression of lipid synthesis-related genes (Table 1 and Fig 2D), which are also regulated by mTORC1 (Yang et al, 2013). Of note, the reduced expression of those genes upon stimulation with TCR plus CGAMP was similarly observed in Raptor-KO T cells upon TCR stimulation (Fig S2F). Recently, it has been shown that mTORC1-induced cholesterol synthesis is critical for antigen-driven clonal expansion (Kidani et al, 2013). LC-mass spectrometry (LC-MS) analysis showed the total content of cholesterol esters was drastically reduced in $T$ cells stimulated with anti-CD3/28 plus CGAMP as compared with $T$ cells stimulated with anti-CD3/28 (Fig 2E). Lipin-1 (Han et al, 2006), the enzyme generating DAG, is a target of, and regulated by, mTORC1 (Eaton et al, 2013). MS analysis demonstrated that the total DAG was substantially reduced in T cells stimulated with TCR and CGAMP (Fig 2E). These data support the idea that the STING-mediated signal inhibits lipid synthesis through the inhibition of TCR-induced mTORC1 activation.

Altogether, STING activation induces the suppression of mTORC1 signaling and results in defective T-cell proliferation.

\section{CDNs induce type I IFN production from T cells in TCR stimulation-dependent manner}

RNA-seq analysis revealed that the top 20 up-regulated genes in T cells upon stimulation with anti-CD3/28 plus CGAMP (Fig S3A) were all interferon-stimulated genes (ISGs) except for an unknown gene, AW011738, suggesting that T cells may induce IFN-I production by STING activation as innate cells. We found that cGAMP alone could induce the expression of IFN-I mRNA (Fig S3C) but failed to produce either IFN- $\beta$ (Fig 3A) or IFN- $\alpha$ (Fig S3B). However, when activated with anti-CD3/CD28, CGAMP and c-di-AMP induced IFN-I production from both naive $\mathrm{CD}^{+}$and $\mathrm{CD} 8^{+} \mathrm{T}$ cells (Figs $3 \mathrm{~A}$ and $\mathrm{S} 3 \mathrm{~B}$ and $\mathrm{S} 3 \mathrm{D}$ ). Interestingly, robust production of type III IFN (IFN- $\lambda 2 / 3$ ) was also observed (Fig S3E). IFN-I production correlated with the signal strength of TCR stimulation (Fig S3F). STING-induced IFN-I
Table 1. Pathway-enrichment analysis.

\begin{tabular}{|c|c|c|}
\hline & Count & $P$ \\
\hline \multicolumn{3}{|l|}{ Enrichment cluster 1 (score, 3.64) } \\
\hline Lipid biosynthesis & 14 & $5.1 \times 10^{-8}$ \\
\hline Lipid metabolic process & 20 & $8.4 \times 10^{-6}$ \\
\hline Lipid metabolism & 18 & $8.9 \times 10^{-6}$ \\
\hline Fatty acid desaturase, type 1 & 5 & $9.4 \times 10^{-6}$ \\
\hline Unsaturated fatty acid biosynthetic process & 5 & $3.3 \times 10^{-5}$ \\
\hline Stearoyl-CoA 9-desaturase activity & 4 & $3.7 \times 10^{-5}$ \\
\hline Fatty acid biosynthesis & 7 & $3.8 \times 10^{-5}$ \\
\hline Fatty acid biosynthetic process & 8 & $4.7 \times 10^{-5}$ \\
\hline Biosynthesis of unsaturated fatty acid & 6 & $5.1 \times 10^{-5}$ \\
\hline Fatty acid metabolism & 9 & $1.0 \times 10^{-4}$ \\
\hline Long-chain fatty acid biosynthetic process & 4 & $1.7 \times 10^{-4}$ \\
\hline PPAR signaling pathway & 8 & $2.3 \times 10^{-4}$ \\
\hline Palmitoyl-CoA 9-desaturase activity & 3 & $9.2 \times 10^{-4}$ \\
\hline Monosaturated fatty acid biosynthetic process & 3 & $9.8 \times 10^{-4}$ \\
\hline \multicolumn{3}{|l|}{ Enrichment cluster 2 (score, 3.42) } \\
\hline Nucleosome & 13 & $1.1 \times 10^{-8}$ \\
\hline Negative regulation of cell proliferation & 14 & $1.5 \times 10^{-3}$ \\
\hline Ubl conjugation & 31 & $2.8 \times 10^{-3}$ \\
\hline \multicolumn{3}{|l|}{ Enrichment cluster 3 (score, 3.31) } \\
\hline ER & 34 & $1.2 \times 10^{-4}$ \\
\hline \multicolumn{3}{|l|}{ Enrichment cluster 4 (score, 3.01) } \\
\hline Sterol biosynthetic process & 6 & $2.2 \times 10^{-5}$ \\
\hline Cholesterol biosynthetic process & 6 & $5.2 \times 10^{-5}$ \\
\hline Cholesterol biosynthesis & 5 & $7.5 \times 10^{-5}$ \\
\hline Steroid biosynthesis & 6 & $7.5 \times 10^{-5}$ \\
\hline Cholesterol metabolism & 6 & $4.5 \times 10^{-4}$ \\
\hline Sterol metabolism & 6 & $7.8 \times 10^{-4}$ \\
\hline Cholesterol metabolic process & 7 & $1.0 \times 10^{-3}$ \\
\hline Steroid biosynthetic process & 6 & $1.2 \times 10^{-3}$ \\
\hline Steroid metabolism & 6 & $1.7 \times 10^{-3}$ \\
\hline \multicolumn{3}{|l|}{ Enrichment cluster 4 (score, 2.26) } \\
\hline Oxidereductase & 19 & $5.3 \times 10^{-4}$ \\
\hline Oxidereductase activity & 19 & $6.0 \times 10^{-4}$ \\
\hline Oxidation-reduction process & 19 & $3.0 \times 10^{-3}$ \\
\hline NADP & 7 & $1.7 \times 10^{-3}$ \\
\hline
\end{tabular}

Pathway-enrichment analysis of gene expression with down-regulation in $\mathrm{CD}^{+} \mathrm{T}$ cells stimulated with anti-CD3/28 plus CGAMP for $24 \mathrm{~h}$ as compared with those stimulated with anti-CD3/28 alone. Data were analyzed with DAVID Bioinformatics Resources 6.8 and represent one experiment.

production in T cells was not induced within $24 \mathrm{~h}$ after stimulation (Fig 3A), although DCS can produce IFN-I within a few hours in response to STING ligands (Roth et al, 2014). Notably, DMXAA could not induce IFN-I production in T cells (Figs 3A and S3B). 
A

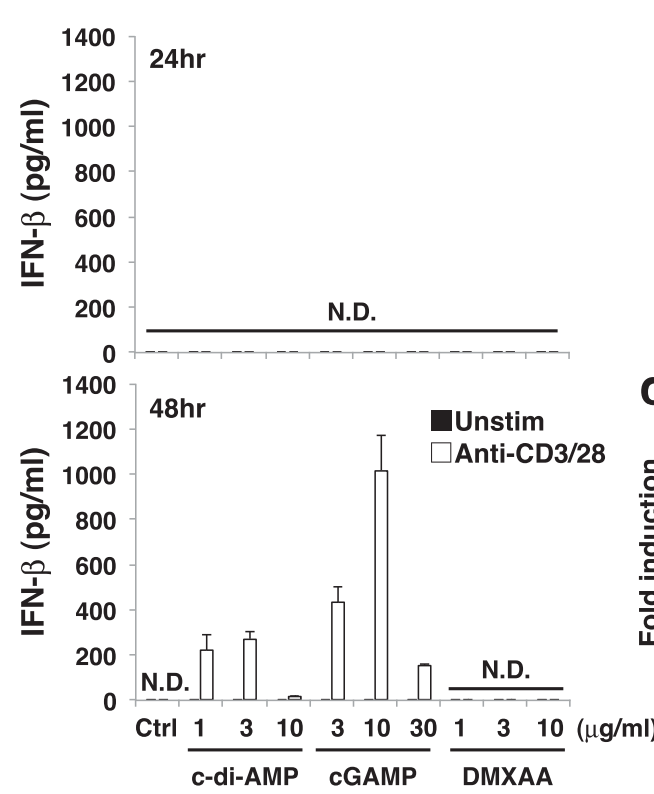

B



C

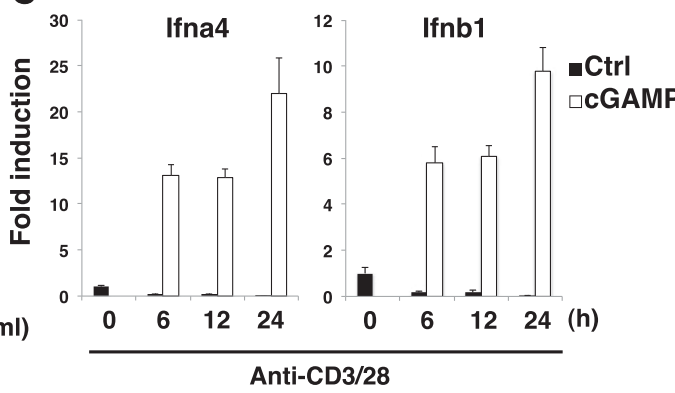

E 1200

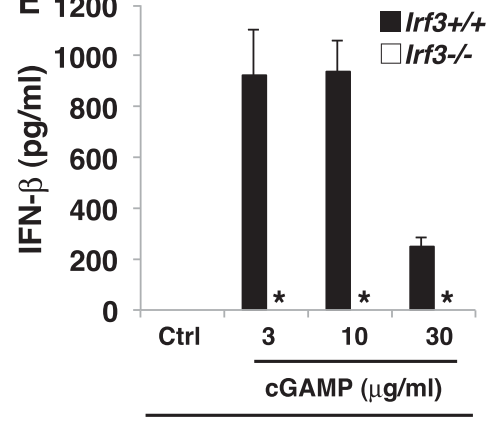

Anti-CD3/28

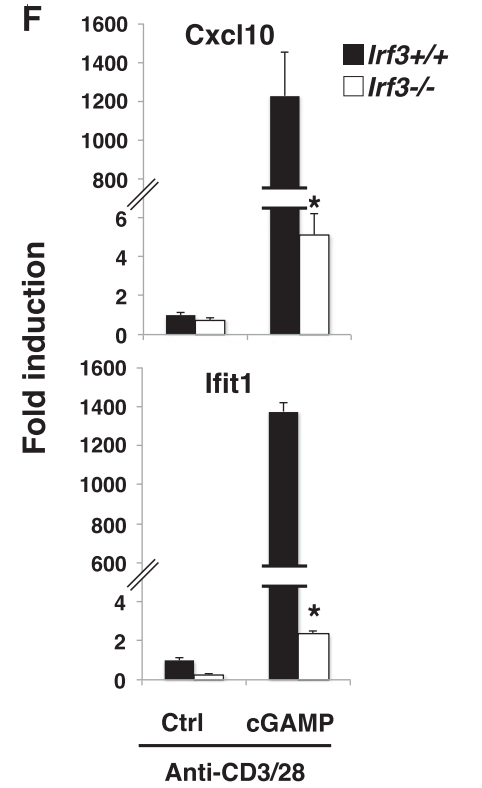

G

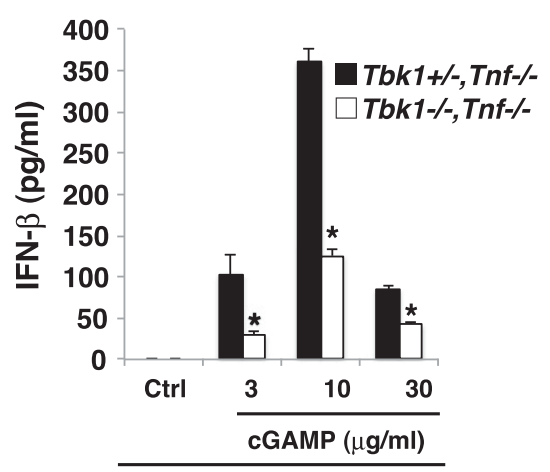

Anti-CD3/28

H

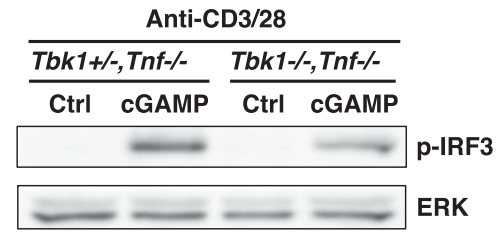
CGAMP DMXAA CGAMP DMXAA

D

$\begin{array}{lllllll}0 & 5 \quad 15 & \overline{5} 15 & \overline{5} 15 & \overline{515} & (\mathrm{~h})\end{array}$
$\square=\square=\square-\square=\square$ ERK

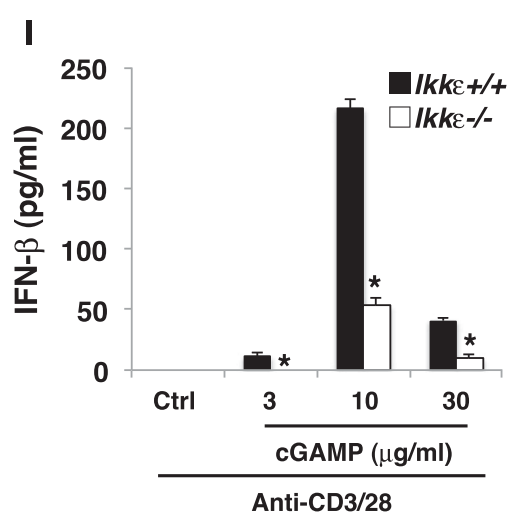

J

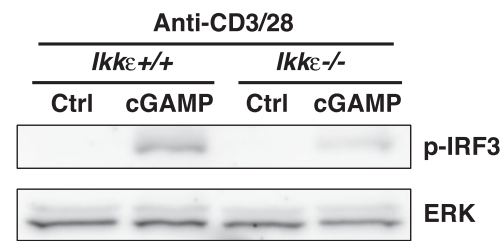

Figure 3. TCR stimulation induces sustained activation of IRF3 upon CDN stimulation to produce type I IFNs.

(A) Naive CD4 $4^{+} \mathrm{T}$ cells were stimulated with anti-CD3/CD28 Abs with or without the indicated STING ligands, and the level of IFN- $\beta$ produced at $24 \mathrm{~h}$ (upper) and $48 \mathrm{~h}$ (lower) after stimulation was measured by ELISA. N.D., not detected $<2.0 \mathrm{pg} / \mathrm{ml}$. (B) Western blot analysis of the activation status in CD ${ }^{+} \mathrm{T}$ cells upon stimulation with cGAMP in the presence or absence of stimulation with anti-CD3/CD28 Abs for 5 or $15 \mathrm{~h}$. (C) qPCR analysis of the expression of IFN genes in CD4 $4^{+}$cells upon stimulation with anti-CD3/CD28 Abs with or without CGAMP for the indicated time period. (D) Western blot analysis for IRF3 activation in CD4 ${ }^{+}$T cells upon stimulation with

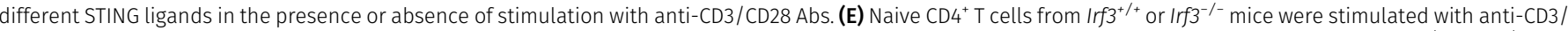
CD28 Abs in the presence or absence of the indicated ligands, and IFN- $\beta$ production was assessed by ELISA. (F) qPCR analysis of ISG genes in CD ${ }^{+}$T cells from Irf3 ${ }^{+/+}$or IIf $3^{-/-}$mice

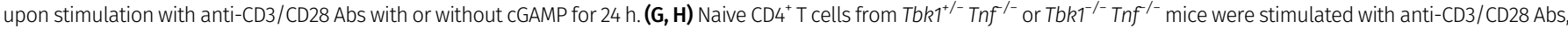


To clarify the mechanism underlying CGAMP-induced IFN-I production upon TCR stimulation, we analyzed the activation of TBK1 and IRF3, which are essential for IFN-I induction in innate cells (Ishii et al, 2006; Takaoka et al, 2007). Whereas cGAMP stimulation alone induced transient phosphorylation of IRF3, CGAMP together with TCR stimulation induced sustained activation of IRF3 and TBK1 (Fig 3B). Consistently, sustained expression of IFN-I mRNA was observed in naive $\mathrm{CD}^{+} \mathrm{T}$ cells stimulated with anti-CD3/28 plus CGAMP (Fig 3C). Although DMXAA activated IRF3 more strongly than CGAMP, the activation was just transient, even in the presence of TCR stimulation (Fig 3D). Together, these data suggest that sustained activation of IRF3 is required for IFN-I production by T cells. It is noted that sustained activation of IRF3 induced by CGAMP and TCR stimulation was observed as early as $15 \mathrm{~h}$ after stimulation (Fig 3B) when T-cell division was not yet induced, indicating that TCRinduced sustained phosphorylation of IRF3 is induced independently of cell division. To confirm the requirement of sustained IRF3 activation for the induction of IFN-I in T cells, IRF3-KO naive CD4 ${ }^{+}$ $T$ cells were stimulated with anti-CD3/CD28 and CGAMP. The induction of IFN-I production and ISGS, such as CXCL10 and IFIT1, was completely eliminated in IRF3-KO CD4 ${ }^{+} \mathrm{T}$ cells (Fig $3 \mathrm{E}$ and F). We next investigated the contribution of TBK1 to IFN-I responses by T cells. Unlike in innate cells, both IFN-I production and IRF3 activation were only partially impaired in TBK1-KO naive $\mathrm{CD}^{+}{ }^{+} \mathrm{T}$ cells (Fig $3 \mathrm{G}$ and $H$ ). Then, we analyzed the contribution of $I K K \varepsilon$, which is a close homologue of TBK1 but only partially contributes to the induction of several ISGs such as CCL5 and CCL2 in innate cells (Ishii et al, 2006). We found that $T$ cells highly express IKKE (Fig S3G) and that both IFN-I production and IRF3 activation were partially reduced in IKKE-KO CD4 ${ }^{+} \mathrm{T}$ cells (Fig $3 \mathrm{I}$ and J). These data suggest that, unlike innate cells, both TBK1 and IKKE are equally and redundantly important for IFN-I production by T cells through the activation of IRF3.

\section{Effector T cells produce robust type I IFNs}

We previously reported that TLR2 ligands directly induce IFN- $\gamma$ production by effector Th1 and $\mathrm{CD}^{+} \mathrm{T}$ cells even without TCR stimulation and that this is enhanced by IL-2 (Imanishi et al, 2007). We assumed a similar possibility that STING ligands might also directly stimulate IFN-I production from effector T cells in the absence of TCR stimulation. As expected, CGAMP induced IFN-I production from Th1 cells and activated $C D 8^{+} T$ cells even in the absence of TCR stimulation, and this was further enhanced by IL-2 and strongly augmented by TCR stimulation (Figs 4A and S4A). Importantly, the amount of IFN-I produced by activated $C D 8^{+} T$ cells upon stimulation with TCR and CGAMP (12 ng/ml, Fig.4A) was 10-fold higher than that from BMDCs (1-2 ng/ml, Fig S4B) (Figs 4A and B, and S4A and B). Notably, unlike naive $T$ cells, effector $T$ cells can produce IFN-I within $24 \mathrm{~h}$ in response to cGAMP. To elucidate the molecular mechanisms underlying direct induction of IFN-I production from effector T cells by CGAMP, we analyzed the signaling molecules downstream of STING in effector
T cells. Whereas CGAMP alone transiently induced the activation of IRF3 in naive T cells (Fig 3B), sustained activation of IRF3 was induced in effector Th1 cells and activated $\mathrm{CD}^{+} \mathrm{T}$ cells (Fig $4 \mathrm{C}$ and D). The activation of IRF3 by CGAMP was augmented by IL-2 or TCR stimulation (Fig 4C and D) and correlated with the IFN-I production. These data indicate that CGAMP alone stimulates IFN-I production from effector/activated T cells by inducing the sustained activation of IRF3, which is further enhanced by IL-2 or TCR stimulation. We also analyzed the other adaptive immune cells, B cells, and found that LPS or anti-IgM plus CGAMP stimulation did not induce IFN-I production by $B$ cells, but CGAMP inhibited LPS or anti-IgM induced B-cell proliferation similarly to TCR-activated T cells (Fig S4C). Unlike in $T$ cells, CGAMP transiently induced expression of IFN-I mRNA upon LPS plus CGAMP stimulation of B cells (Fig S4D).

\section{IRF3/7 are required for the STING-mediated inhibition of mTORC1 and IL-2 signaling}

Because T cells can produce IFN-I in response to CGAMP and TCR stimulation, it is possible that IFN-I produced by the T cells may inhibit the activation of mTORC 1 and the proliferation. This issue was addressed by analyzing these signals in IFN $\alpha$ receptor 1 (IFNAR1)-KO $\mathrm{CD}^{+} \mathrm{T}$ cells. Phosphorylation of S6K1, 4E-BP1, and STAT5 was similarly inhibited by CGAMP, whereas CGAMP-induced growth inhibition was partially restored in IFNAR1-KO T cells (Fig 5A and B), indicating that IFN-I signaling is partly involved in growth inhibition but not in inhibition of mTORC1 and IL-2 signaling pathways. We confirmed that cGAMP-induced growth inhibition was also partly blocked in the presence of rapamycin, an mTOR inhibitor (Fig S5A), indicating that both the inhibition of mTORC1 activation and IFN-I signaling are involved in STING-mediated growth inhibition. In addition, it is noted that the simultaneous treatment with both rapamycin and antiIFNAR1 blocking Ab did not completely block the cGAMP-induced growth inhibition (Fig S5A), suggesting that yet unidentified pathway is involved in STING-mediated growth inhibition.

Next, we sought to determine the mechanism of STING-mediated inhibition of both mTORC1 and IL-2 pathways. We first examined the involvement of TBK1 and IKKE in the CGAMP-induced inhibition of mTORC1 and IL-2 pathways. Surprisingly, CGAMP-induced growth inhibition was equivalent in $\mathrm{CD}^{+} \mathrm{T}$ cells from TBK1- and IKKE-KO mice (Fig 5C). We then analyzed the involvement of IRF3/7 in this process. CGAMP-induced growth inhibition was partially restored in IRF3-KO CD4 ${ }^{+} \mathrm{T}$ cells (Fig 5C) and in IRF7-KO CD4 ${ }^{+} \mathrm{T}$ cells (Fig S5B) and more strongly restored in IRF3/7-doubly deficient (DKO) $\mathrm{CD}^{+}{ }^{+} \mathrm{T}$ cells (Fig 5D). In addition, partial restoration of CGAMP-induced inhibition of mTORC1 (p-S6K1 and p-4E-BP1) and IL-2 (p-STAT5) signaling was observed in IRF3/7-DKO CD4 ${ }^{+} \mathrm{T}$ cells (Figs 5E and S5C). Consistently, the inhibition of the expression of lipid synthesis-related genes, CD98 and CD71, by CGAMP was partly recovered in IRF3/7-DKO CD ${ }^{+}$ $T$ cells (Figs $5 \mathrm{~F}$ and $\mathrm{S} 5 \mathrm{D}$ ). Particularly, the partial restoration of cGAMP-induced growth inhibition in IRF3-KO CD4 ${ }^{+} \mathrm{T}$ cells was

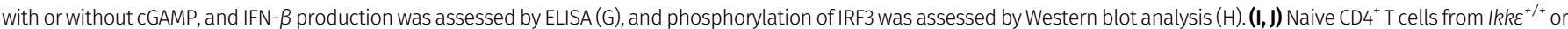
$\mathrm{Ikk \varepsilon}^{-/-}$mice were stimulated with anti-CD3/CD28 Abs with or without CGAMP, and IFN- $\beta$ production was assessed by ELISA (I), and phosphorylation of IRF3 was assessed by Western blot analysis (J). Data are the mean from duplicate $(A, E, G, I)$ or triplicate $(C, F) \pm S D$. Data are representative of at least three independent experiments. $(A-J) * P<0.05, t$ test $($ compared with WT cells treated with CGAMP). 

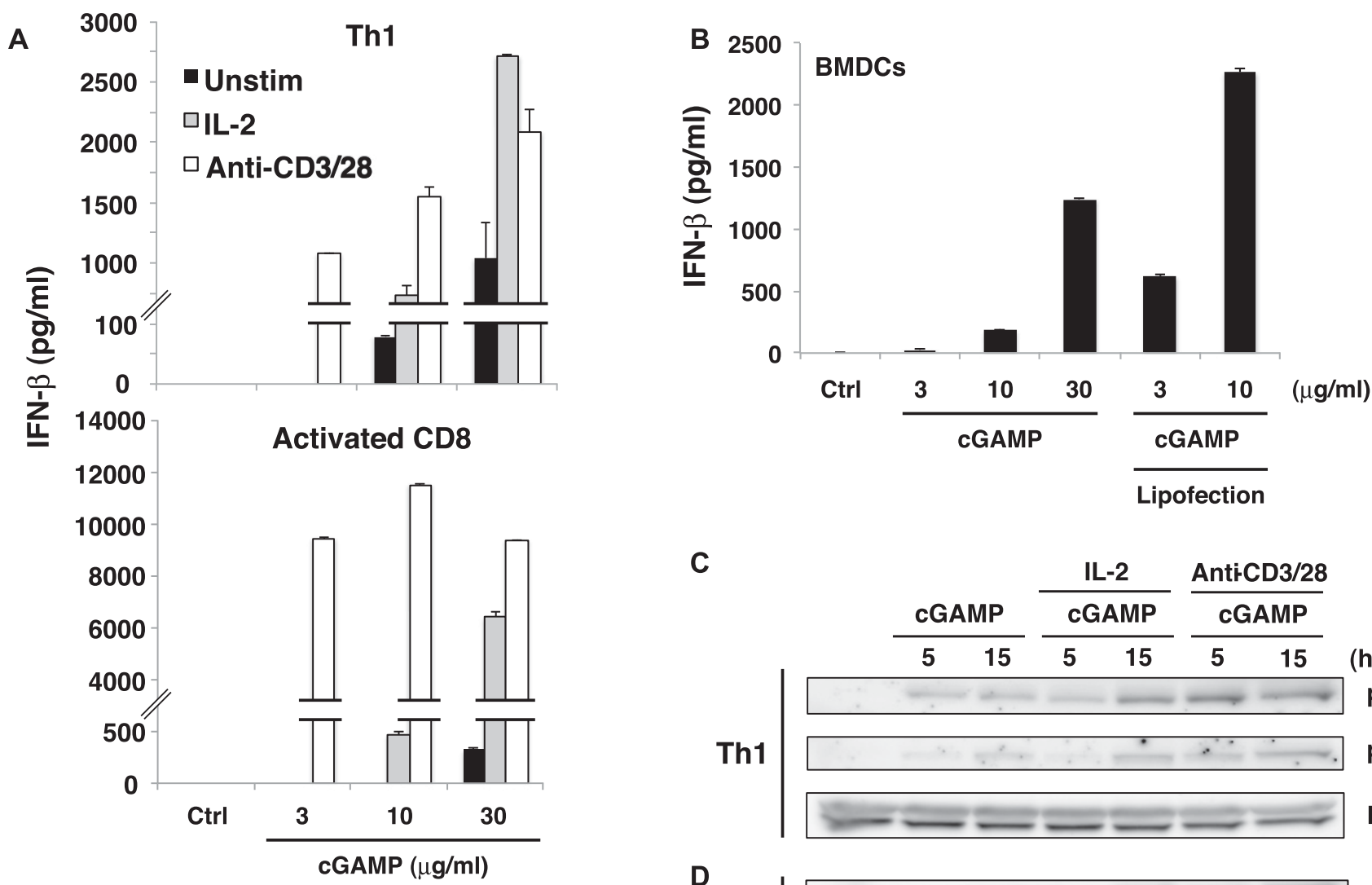

C
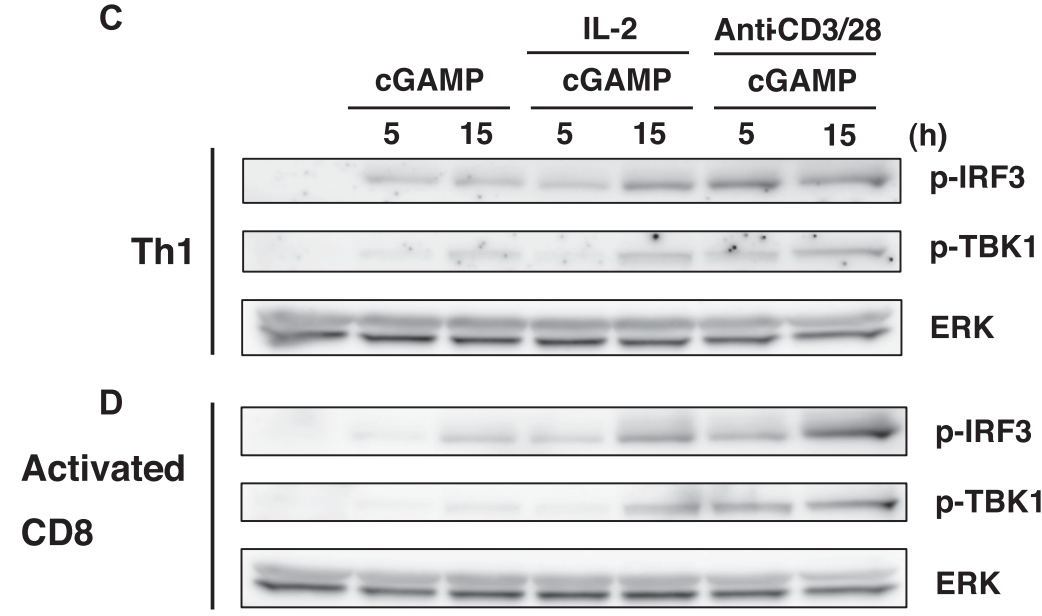

p-IRF3

p-TBK1

ERK

Figure 4. Greatly enhanced STING-mediated type I IFN responses in effector/activated T cells.

(A) Th1 cells or activated $C D 8^{+} T$ cells were stimulated with cGAMP in the presence (IL-2) or absence (unstim) of IL-2 or stimulation with anti-CD3/CD28 Abs for $24 \mathrm{~h}$, and IFN- $\beta$ production was assessed by ELISA. (B) CGAMP was added to the media or introduced by lipofection into BMDCS for $24 \mathrm{~h}$, and IFN- $\beta$ production was assessed by ELISA. (C, D) Western blot analysis of phosphorylation of IRF3 and TBK1 in Th1 cells (C) or activated CD8 ${ }^{+}$T cells (D) upon stimulation with cGAMP with or without $\mathrm{IL}-2$ or anti-CD3/CD28 stimulation. Data are the mean from duplicate $\pm \mathrm{SD}(\mathrm{A}, \mathrm{B})$. Data are representative of at least three independent experiments $(A-D)$.

completely cancelled by the addition of exogenous IFN- $\beta$ (Fig S5E), whereas the addition of exogenous IFN- $\beta$ partially cancelled it in IRF3/7-DKO CD4 ${ }^{+} \mathrm{T}$ cells (Fig S5F). In addition, CGAMP-induced inhibition of mTORC1 activation was largely intact in IRF3-KO CD4 ${ }^{+}$ T cells (Fig S5G), suggesting that IRF3 and IRF7 have redundant function in STING-mediated T-cell growth inhibition. These data also suggest that normal cGAMP-induced growth inhibition in TBK1or IKKE-KO T cells may be due to the remaining IFN-I production because CGAMP-induced IFN-I production was completely diminished in IRF3-KO T cells (Fig 3E) but only partially impaired in TBK1- or IKKE-KO T cells (Fig 3G and I).

We also analyzed whether cGAMP treatment may affect ER stress pathways because it has been shown that STING departure from ER causes ER stress that inhibits mTOR pathway (Moretti et al, 2017). However, phosphorylation of PERK, IRE1 $\alpha$, and eIF2 $\alpha$, which represent ER stress transducers, was not altered by cGAMP treatment (Fig $\mathrm{S} 5 \mathrm{H}$ ), indicating that inhibition of mTOR was not induced by ER stress but by STING signals in T cells.
Collectively, these data indicate that the TBK1/IKKE-IRF3/7-IFN-I axis inhibits $\mathrm{T}$-cell proliferation and IRF3/7 are also critical for STING-mediated inhibition of MTORC1 and IL-2 pathways independently of TBK1/IKKE.

\section{TCR-induced activation of mTORC1 is required for STING- mediated type I IFN production}

Although our data verified that the STING-IRF3/7 axis inhibits T-cell proliferation through blocking mTORC1 function, the involvement of mTORC1 in STING-mediated IFN-I production remains unclear. When naive $\mathrm{CD}^{+} \mathrm{T}$ cells were stimulated with anti-CD3/CD28 plus CGAMP in the presence of rapamycin, surprisingly, CGAMP-induced IFN-I production and the expression of ISGs were completely abrogated (Figs S6A and 6B). To confirm the importance of mTORC1 in STING-mediated IFN-I responses, we analyzed Raptor-KO $\mathrm{CD}^{+}$ $T$ cells because Raptor is an essential component of the mTORC1 complex. Similar to rapamycin-treatment, cGAMP-induced IFN-I 
A

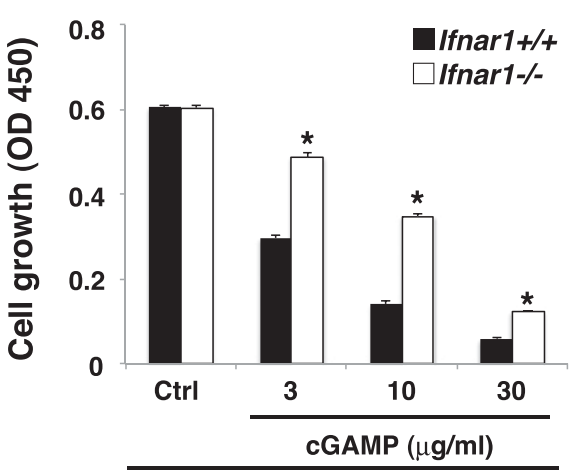

Anti-CD3/28
B

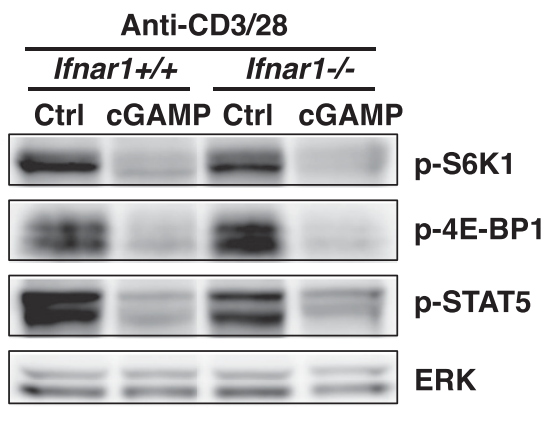

E

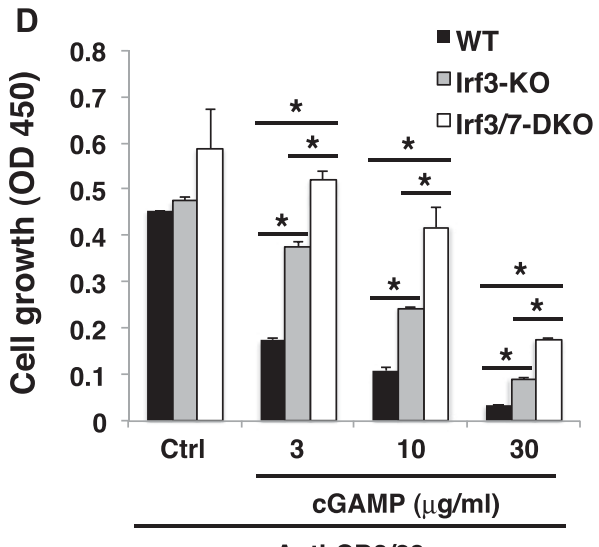

$\mathbf{F}$
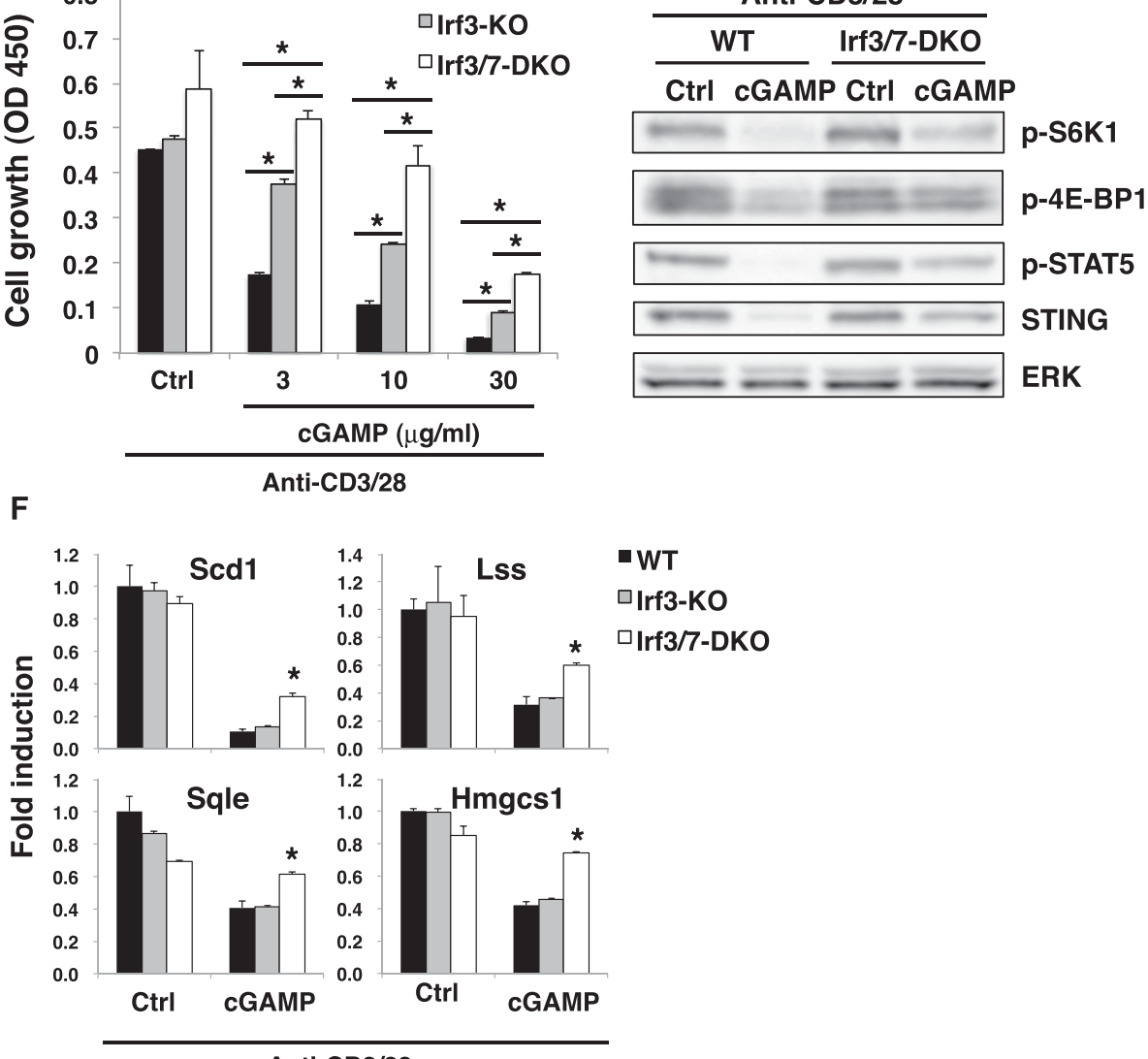

Anti-CD3/28
C

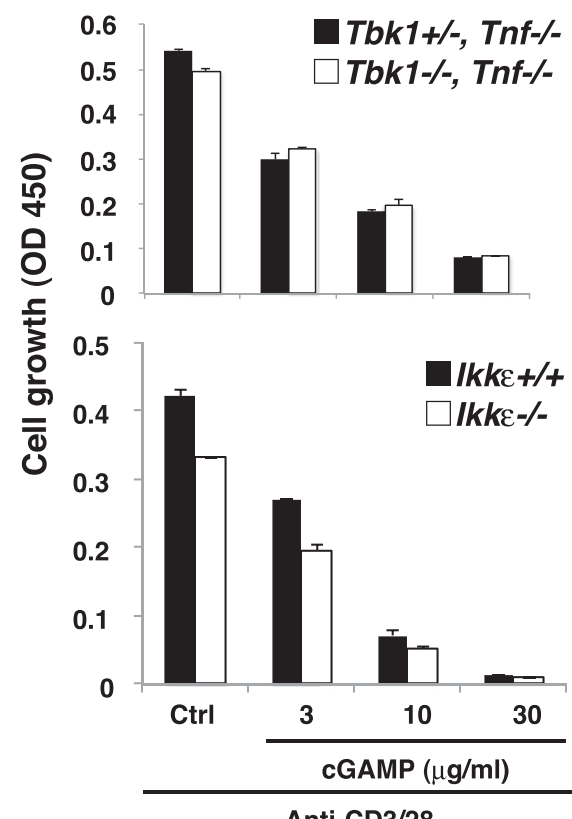

Anti-CD3/28

Figure 5. Molecular mechanism of STING-mediated inhibition of the mTORC1 pathway.

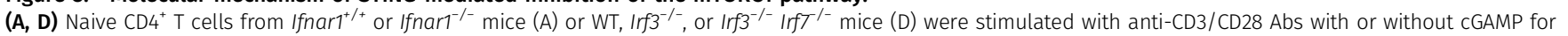
$48 \mathrm{~h}$, and cell growth was assessed by a WST-8 cell proliferation assay. (B, E) Activation of the mTORC1 pathway was analyzed by Western blot in CD $4^{+} \mathrm{T}$ cells from Ifnar1 ${ }^{+/ *}$

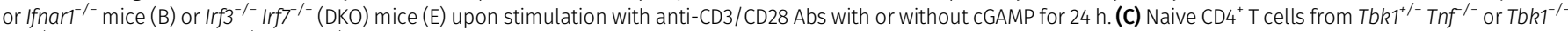

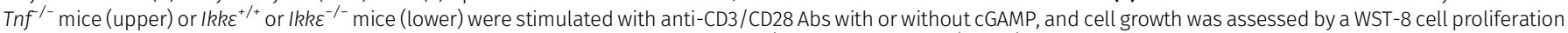
assay. (F) qPCR analysis of various genes in $\mathrm{CD}^{+} \mathrm{T}$ cells from WT (closed bar), Irf3 ${ }^{-/-}$(grey bar), or Irf3 $3^{-/-}$Irf $7^{-/-}$(open bar) mice upon stimulation with anti-CD3/CD28 Abs with or without CGAMP for $24 \mathrm{~h}$. Data are the mean from duplicate \pm SD $(A, C, D)$ or triplicate $(F) \pm S D$. Data are representative of at least three independent experiments $(C-F)$. $(\mathbf{A}, \mathbf{F}){ }^{*} P<0.05, t$ test (compared with WT cells treated with cGAMP). (D) ${ }^{*} P<0.05$, $t$ test (compared with WT cells or Irf3-KO cells treated with cGAMP).

production was completely abrogated in Raptor-KO $\mathrm{CD}^{+} \mathrm{T}$ cells (Fig 6A). Quantitative PCR ( $\mathrm{PPCR}$ ) analysis revealed that the expression of type I and type III IFNs and ISGs, except for CCl5, was severely impaired in Raptor-KO T cells (Fig 6B) and by rapamycin treatment
(Fig S6B), suggesting that mTORC1 signaling specifically regulates the induction of STING-mediated genes. Because TCR stimulation strongly activates the mTORC1 pathway and induces the sustained phosphorylation of IRF3, which is essential for the induction of IFN-I 

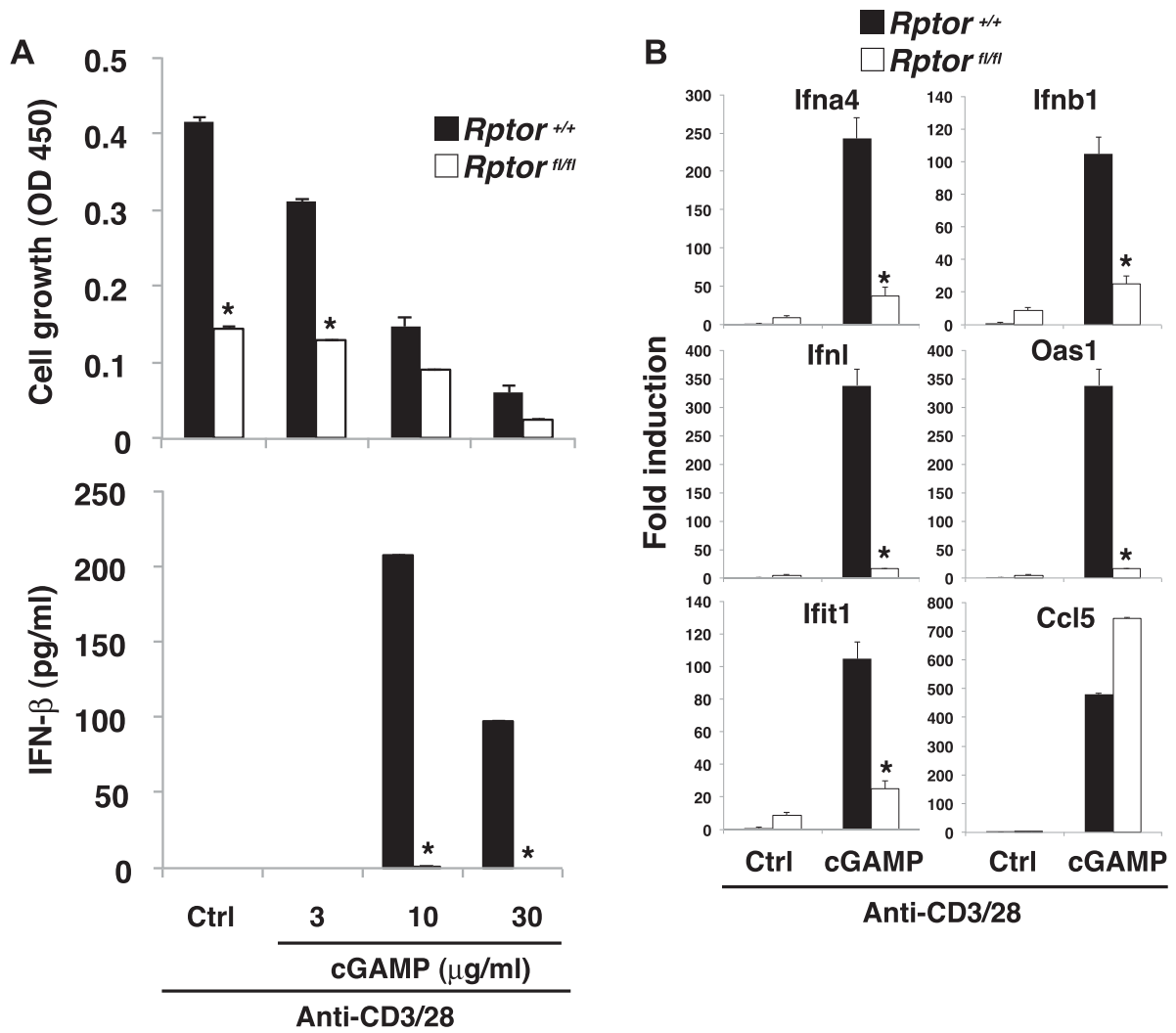

C


Anti-CD3/28

Figure 6. mTORC1 signaling is required for STING-mediated type I IFN responses.

(A) Naive $\mathrm{CD}^{+} \mathrm{T}$ cells from Rptor ${ }^{++}$(closed bar) or Rptorfl/fl (open bar) mice were stimulated with anti-CD3/CD28 Abs with or without cGAMP for $48 \mathrm{~h}$, and cell growth (upper) and IFN- $\beta$ production (lower) were assessed by a WST-8 cell proliferation assay and ELISA, respectively. (B) qPCR analysis of ISG genes in CD4 ${ }^{+} T$ cells from Rptor ${ }^{+/+}$ (closed bar) or Rptorlf/fl (open bar) mice upon stimulation with anti-CD3/CD28 Abs with or without CGAMP for 24 h. (C) Western blot analysis of activation of IRF3 and mTORC1-related molecules in CD4 ${ }^{+} \mathrm{T}$ cells with or without rapamycin pretreatment and upon stimulation with anti-CD3/CD28 Abs with or without cGAMP for $24 \mathrm{~h}$ (upper). Relative intensity of IRF7 was shown (lower). (D) Naive CD4 ${ }^{+} \mathrm{T}$ cells were untreated or pretreated with S6K inhibitor PF-4708671 and stimulated with anti-CD3/CD28 Abs with or without CGAMP for $48 \mathrm{~h}$, and IFN- $\beta$ production was assessed by ELISA. N.D., not detected $<2.0 \mathrm{pg} / \mathrm{ml}$. (E) CD4 ${ }^{+} \mathrm{T}$ cells were retrovirally transduced with an empty vector (EV) or a $4 \mathrm{E}-\mathrm{BP} 1$ (T37/46 A/A) construct and stimulated with anti-CD3/CD28 Abs with or without CGAMP for $24 \mathrm{~h}$, and IFN- $\beta$ production was assessed by ELISA. Data are the mean from duplicate (A, D, E) or triplicate $(B) \pm S D$. Data are representative of at least three independent experiments (C-E). (A, B) ${ }^{*} P<0.05, t$ test (compared with WT cells treated with cGAMP). (D) ${ }^{*} P<0.05, t$ test (compared with that with anti-CD3/28 alone). (E) ${ }^{*} P<0.05, t$ test (compared with control cells treated with CGAMP).

and ISGs by cGAMP (Figs 3B, 3E, and F), we assumed that TCRinduced mTORC1 activation may be critical for the sustained phosphorylation of IRF3. However, rapamycin did not alter IRF3 activation by cGAMP plus TCR stimulation in $\mathrm{CD}^{+} \mathrm{T}$ cells (Fig 6C), indicating that TCR-induced mTORC1 signaling is critical for STINGmediated induction of IFN-I and ISGs independently of IRF3 
activation. It is noted that the inhibition of T-cell growth by rapamycin treatment was similarly induced to the simultaneous treatment with both rapamycin and anti-IFNAR1 blocking Ab (Fig $\mathrm{S} 5 \mathrm{~A}$ ) because rapamycin treatment inhibited both mTORC1 activation and CGAMP-induced IFN-I production.

In that case, how does mTORC1 signaling affect STING-mediated IFN-I responses? It has been reported that $4 \mathrm{E}-\mathrm{BP} 1 / 2$ negatively regulates IFN-I production via translational repression of IRF7 mRNA in innate cells (Colina et al, 2008). In fact, IRF7 protein expression was slightly reduced in $\mathrm{CD}^{+} \mathrm{T}$ cells treated with rapamycin (Fig $6 \mathrm{C}$ ), suggesting that mTORC1 is critical for IFN-I production by $T$ cells stimulated with anti-CD3/28 plus cGAMP partly at least through translational control of IRF7. Then, we analyzed the involvement of S6K1 and 4E-BP1 as the main targets of mTORC1 in STING-mediated IFN-I production. PF-4708671, a specific inhibitor of S6K1 (Pearce et al, 2010), inhibited CGAMP-induced IFN-I production by $C D 4^{+} T$ cells upon TCR stimulation in a dose-dependent manner (Fig 6D). We also found that $T$ cells overexpressing the dominant-negative 4E-BP1 (Thr-37/46 to alanine) produced less IFN-I (Fig 6E), indicating that both axes of mTORC1-S6K1 and mTORC1-4E-BP1 are critically involved in STINGmediated IFN-I responses in T cells upon TCR stimulation.

Although CGAMP inhibits mTORC1 activation, which is required for IFN-I production by T cells, CGAMP induces but does not inhibit IFN-I production. This paradox appears to be regulated by a quantitative balance between STING and mTORC1 signaling. Increasing doses of cGAMP induce stronger activation of IRF3 and higher production of IFN-I (Fig S6D and E). By contrast, the activation of the mTORC1 pathway, 4E-BP1 and S6K1, was inhibited by CGAMP in a dose-dependent manner (Fig S6E). 4E-BP1 was strongly inhibited, whereas S6K1 was weakly inhibited by CGAMP as compared with rapamycin (Fig S6C), suggesting that the S6K1 pathway may contribute to the induction of IFN-I production. Consistently, rapamycin completely inhibited IFN-I production through complete inhibition of both 4E-BP1 and S6K1 phosphorylation, whereas CGAMP mediated relatively weak inhibition of S6K1 (Fig S6F). In addition, the week inhibition of S6K1 activation by treatment with $0.1 \mathrm{nM}$ rapamycin partially inhibited CGAMP-induced IFN-I production, whereas the same treatment inhibited T-cell proliferation as strongly as higher concentrations of rapamycin (Fig S6G). Therefore, it is likely that partial inhibition of S6K1 pathway by CGAMP allows the CGAMPinduced IFN-I production through the remaining activity of S6K1 and the downstream molecules of S6K1. Together, these findings indicate that activation of STING induces the partial inhibition of
mTORC1 activation, which is sufficient to inhibit T-cell proliferation on the one hand, whereas this partially remained activation of mTORC1 signals is critical for IFN-I production on the other hand (Fig S7).

\section{Critical role for STING in T cells in antitumor responses}

It has been recently demonstrated that activation of the STING pathway is critical for antitumor immune responses in vivo (Woo et al, 2014; Deng et al, 2014). Indeed, STING-KO mice show impaired antitumor responses to radiation and immune checkpoint blockade therapies, such as PD-1/PD-L1 and CTLA4 (Woo et al, 2014; Deng et al, 2014; Demaria et al, 2015; Wang et al, 2017). Administration of STING ligands inhibits tumor growth and potentiates the antitumor effects of radiation and immune checkpoint blockade through the production of IFN-I (Deng et al, 2014; Demaria et al, 2015; Temizoz et al, 2015). Our finding that activated $C D 8^{+} T$ cells produce much higher levels of IFN-I than innate cells such as BMDCs (Fig 4A) raises the possibility that STING expressed in T cells may contribute to antitumor immune responses. To test this possibility, we made T cell-specific STING-KO mice by reconstituting RAG1-KO mice with STING-KO T cells and WT B cells. The mice were then inoculated with B16 melanoma cells, followed by injection of CGAMP intratumorally on day 8,10 , and 13 after tumor inoculation, and the tumor growth and survival were monitored. Tumor growth was accelerated in T cell-specific STING-KO (Sting ${ }^{-/}$) mice as compared with control mice $\left(\right.$ Sting $\left.{ }^{+/-}\right)$, which had received both Sting ${ }^{+-}$T cells and WT $B$ cells (Fig 7A). Consistently, the survival of the tumor-bearing $\mathrm{T}$ cell-specific STING-KO mice were significantly lower than the control mice (Fig 7B).

These results suggest that STING expressed in T cells plays a crucial role in antitumor immunity.

\section{Discussion}

Our studies have demonstrated that STING activation in T cells induces the suppression of T-cell proliferation through the inhibition of the mTORC1 pathway and the IFN-I signaling. This is the first report showing a functional link between the STING pathway and MTOR, the metabolic checkpoint kinase. STING-mediated inhibition of the mTORC1 pathway may be beneficial for host defense because the inhibition of pathogen-infected T-cell
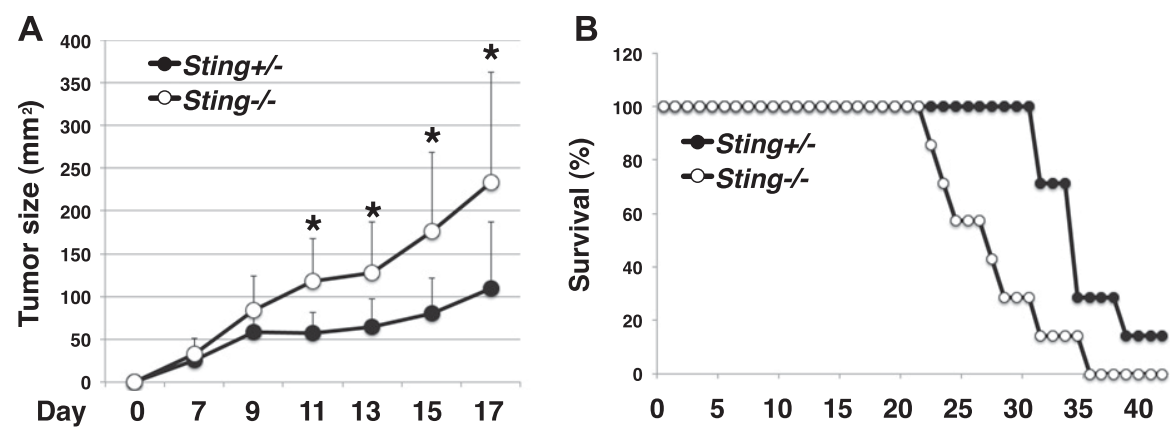

Figure 7. T cell-intrinsic STING is critical for antitumor immunity.

(A, B) T cells from Sting ${ }^{+/-}$or Sting $^{-/-}$mice and B220+ cells from WT mice were cotransferred into Rag1-KO mice $\left(\right.$ Sting $^{+/-}$, Sting $\left.^{-/-}\right)$, and the mice were subcutaneously inoculated with $2 \times 10^{5}$ B16 F10 melanoma cells ( $n=7$ mice per group). On days 8,10 , and 13 , the mice were subjected to intratumor injection of CGAMP and then monitored for tumor growth for $17 \mathrm{~d}(\mathrm{~A})$ and for survival for $40 \mathrm{~d}$ (B). (A) ${ }^{*} P<0.05, t$ test. 
growth could block pathogen replication. Viruses have evolved to modify various cell signaling pathways in the host to establish optimal environments for their replication and spread. Most viruses induce glycolysis, fatty acid synthesis, and glutaminolysis in host cells to increase the energy supply for their replication (Sanchez \& Lagunoff, 2015). mTORC1 is critical for TCR-induced glycolysis and fatty acid synthesis, and glutaminolysis activates the mTORC1 pathway (Duran et al, 2012). Therefore, STINGmediated inhibition of mTORC1 might be detrimental to virus replication and thus an adaptive host strategy to suppress virus replication.

Our data show that STING-mediated inhibition of mTORC1 partly requires IRF3/7 but not TBK1/IKKE in T cells. Because TBK1/IKKEmediated phosphorylation of IRF3/7 is required for the induction of IFN-I genes in T cells, it is possible that IRF3/7 may inhibit the activation of mTORC1 independently of their function as transcription factors. Such function of IRF3 has been reported that IRF3 induces viral apoptosis through the interaction of its BH3-like domain with the pro-apoptotic protein Bax (Chattopadhyay et al, 2010). In addition, it has been reported that IRF3-mediated apoptosis requires linear polyubiquitination of IRF3 by LUBAC through TRAFs but not TBK1 (Chattopadhyay et al, 2016). Therefore, it is likely that IRF3/7 have a unique function other than transcription factors to suppress the mTORC 1 activation in T cells because TBK1, a transcriptional regulator for IRF3, is not involved in STING-mediated inhibition of mTORC1 pathway. The mechanism of IRF3/7 to inhibit mTORC1 remains to be further investigated. Because STING-mediated mTORC1 inhibition was not completely restored in IRF3/7-DKO T cells, other molecules including other members of the IRF family, for example, IRF1, 5 and 9, may have functional redundancy with IRF3/7.

The most surprising finding in this study was that $T$ cells produce IFN-I upon stimulation with STING and TCR, because T and B cells have been believed not to produce IFN-I. STING ligands induce robust IFN-I production by T cells similarly to innate cells. A most critical observation was that IFN-I production requires TCR stimulation. Our data clearly demonstrated that the activation of both IRF3 and mTORC1 is essential for STING-mediated IFN-I production. Because TCR stimulation triggers two events in this pathway, induction of the sustained activation of IRF3, and the activation of the mTORC1 pathway, TCR signaling is required to induce IFN-I responses upon STING activation. The reason why STING-mediated IFN-I production from naive T cells is induced later than innate immune cells is because mTOR activation is required for IFN-I production in T cells. mTORC1 activation is induced at the peaks later than $24 \mathrm{~h}$ after TCR stimulation. Consistently, activated effector T cells, in which mTORC1 pathway is already activated, produce type IFN-I shortly within $24 \mathrm{~h}$ in response to CGAMP. Because neither TCR stimulation nor STING activation alone induces IFN-I production, this regulation system allows only antigen-specific activated $T$ cells to produce IFN-I. IFN-I has been shown to exhibit blocking functions in infectious and autoimmune diseases and cancer development. Therefore, IFN-I specifically produced by antigen-specific T cells may play roles in preventing the development of these diseases at inflammatory sites.

We defined here for the first time the reciprocal regulation between STING and the mTORC1 pathway for the modulation of
T-cell functions, particularly induction of growth arrest and IFN-I production. STING-mediated mTORC1 activation together with TCR stimulation induced growth inhibition on the one hand and IFN-I production on the other hand. Because complete blockade of mTOR function by rapamycin completely inhibits IFN-I production, STING ligand-induced inhibition of mTORC1 is not complete with the remaining signals through $\mathrm{S6K1}$, which we found contributes the induction of IFN-I production.

We have demonstrated that STING-mediated responses in T cells vary depending on species and doses of different STING agonists. Indeed, CGAMP and c-di-AMP as CDNs, but not DMXAA, induced IFN-I production upon T-cell stimulation. Conversely, DMXAA but not cGAMP and c-di-AMP induces T-cell death. Consistently, a recent study reported that activation of STING in T cells by DMXAA induces cell death (Larkin et al, 2017). Moreover, even among CDNs, we found that cGAMP induces more IFN-I production than c-di-AMP. Recent studies demonstrated that natural variant alleles of STING, namely, the R232H variant of human STING and the R231A variant of mouse STING, were activated by cGAMP but not c-di-GMP (Diner et al, 2013). Furthermore, it has been reported that DMXAA, which specifically binds to mouse STING, also activates human STING bearing a unique point mutation (S162A) at the CDN-binding site (Gao et al, 2013). These findings suggest that different STING agonists bind to different sites of STING to activate downstream signaling. Therefore, the effect of DMXAA on T cells may be quite different from that of cGAMP and c-di-AMP. Recently, it has been reported that the cellpermeable small molecule STING agonist 10-carboxymethyl-9acridanone (CMA) induces T-cell apoptosis, whereas CMA does not induce IFN-I production (Gulen et al, 2017), suggesting the possibility that the binding site of CMA to STING may be similar to DMXAA. Indeed, CMA and DMXAA are structurally similar, and CMA activates IFN-I responses in murine cells but not human cells such as DMXAA (Cavlar et al, 2013). It has been reported that the duration and the magnitude of FCRY signals determine mast cell survival and degranulation, respectively (Yamasaki et al, 2004). Prolonged ERK activation induced by antigen alone induces survival but not degranulation. By contrast, transient strong ERK activation induced by IgE plus antigen induces degranulation but not survival. Therefore, it is possible that different STING agonists induce different activation status of STING, leading to different outcomes. Considering the different characteristics of the ligands, the development of new STING agonists and antagonists with the best activity as vaccines or IFN-I inducers for immunotherapy of cancer and autoimmune disorders could be achieved.

cGAMP has been shown to provide strong antitumor effects in immune-competent mice. It has been thought that cGAMPenhanced cross-presentation of tumor-associated antigens in DCs to CD8 ${ }^{+}$cytotoxic T cells is one of the mechanisms underlying STING ligand-induced antitumor immunity (Wang et al, 2017). STING is required for radiation-induced antitumor T-cell responses, which are dependent on IFN-I signaling in DCs (Deng et al, 2014). Furthermore, immune-checkpoint therapy using anti-PD-1Ab is effective in the presence of STING activation (Woo et al, 2014; Demaria et al, 2015; Wang et al, 2017). In the present study, we showed that T cell-intrinsic STING is important for the induction of cGAMPinduced antitumor effects. It has been reported that IRF7/IFN- $\beta$ activation enhances chimeric antigen receptors (CARs) T cell-mediated 
antitumor activity (Zhao et al, 2015), suggesting that in addition to IFN-I from innate cells, as is widely believed, STING-mediated production of IFN-I by $T$ cells might contribute significantly to antitumor immunity. It is worth considering that antigen-specific effector $T$ cells are localized in the tumor microenvironment, where the effector cells receive STING activation signals and produce much higher levels of IFN-I than innate cells.

Recently, it has been reported that human T cells transduced with a STING mutant from patients carrying the constitutively active mutation showed reduced proliferation and the patients showed an altered proportion of peripheral T-cell compartments characterized by increased naive T cells and reduced memory-type T-cell populations (Cerboni et al, 2017). Interestingly, T cell-specific Raptor-KO mice also showed reduced memory-type T-cell populations (Yang et al, 2013), suggesting the possibility that the activation of mTORC1 in these patients with activating STING mutations may be impaired.

In summary, our data demonstrate that T cell-intrinsic STING signaling and TCR signaling are coregulated to modulate T-cell functions. Our study has identified a key role of mTORC1-mediated signaling for both STING-mediated growth inhibition and IFN-I responses. These observations could have implications for the development of new therapeutic strategies for cancer, infectious diseases, and autoimmune diseases.

\section{Materials and Methods}

\section{Mice}

C57BL/ 6 mice were purchased from Clea Japan, Inc. Mice deficient in Tbk1, Tnf, and Ikk- $\varepsilon$ were kindly provided by S Akira (Osaka University). IRF3-KO, IRF7-KO, and IRF3/7-DKO mice were provided by $T$ Taniguchi (Tokyo University). Rip3 ${ }^{-/-}$mice were kindly provided by Genentech, Inc. Sting ${ }^{-/-}$and Ifnar- ${ }^{-/-}$mice were kindly provided by GN Barber (University of Miami) and K Miyake (Tokyo University), respectively. Rptor ${ }^{f l / f l}$ mice crossed with Lck-Cre mice were kindly provided by S Matsuda (Kansai Medical University). 6-16-wk-old mice were used. All mice were maintained under specific pathogen-free conditions at RIKEN, and all experiments were conducted under protocols approved by RIKEN Yokohama Institute.

\section{Cell preparation}

$\mathrm{CD}^{+}$and $\mathrm{CD} 8^{+}$naive $\mathrm{T}$ cells were purified from spleen and lymph nodes as $\mathrm{CD}^{+} / \mathrm{CD} 25^{-} / \mathrm{NK} 1.1^{-} / \mathrm{CD} 44^{\text {low }} / \mathrm{CD}_{2} \mathrm{~L}^{\text {high }}$ cells by sorting using FACSAria (BD Biosciences). Th1 effector cells were prepared by stimulation of $\mathrm{CD}^{+} \mathrm{T}$ cells with anti-CD3/CD28 Abs and cultured in the presence of IL-2 (10 ng/ml), IL-12 (10 ng/ml), and anti-IL-4 Ab $(10 \mathrm{ng} / \mathrm{ml})$ for $6 \mathrm{~d}$ in RPMI1640 medium supplemented with 10\% FCS. Activated $\mathrm{CD}^{+} \mathrm{T}$ cells were prepared by stimulating $\mathrm{CD} 8^{+} / \mathrm{CD} 25^{-} /$ NK1.1 $1^{-} /$CD44 ${ }^{\text {low }} /$ CD62 $^{\text {high }}$ (naive) T cells sorted by FACSAria with plate-bound anti-CD3E (2C11, $10 \mu \mathrm{g} / \mathrm{ml})$ and anti-CD28 (PV-1, $10 \mu \mathrm{g} /$ $\mathrm{ml}$ ) (anti-CD3/CD28) and then cultured in the presence of $\mathrm{IL}-2$ (10 $\mathrm{ng} / \mathrm{ml}$ ).
B cells were prepared by sorting $B 220^{+}$cells from splenocytes. BMDCs were prepared by culturing bone marrow cells in the presence of IL-3 and sorted for CD11C ${ }^{+}$cells by FACSAria.

\section{Functional analyses}

T cells were stimulated with immobilized anti-CD3E $(2 \mathrm{C} 11,10 \mu \mathrm{g} / \mathrm{ml})$ and anti-CD28 (PV-1, $10 \mu \mathrm{g} / \mathrm{ml}$ ) Ab with or without STING ligands. For antigen-specific activation of $\mathrm{T}$ cells, $\mathrm{CD}^{+} \mathrm{T}$ cells from OVA-specific TCR-Tg mice OT-II were stimulated by coculturing with T cell-

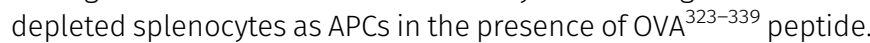
Culture supernatants from these cultures were analyzed by ELISA for production of IL-2 (BD Biosciences), IFN- $\alpha$ (PBL assay science), IFN- $\beta$ (PBL assay science), and IFN- $\lambda 2 / 3$ (PBL assay science). Cell growth was assessed using a Cell Counting Kit-8 (DOJINDO). For apoptosis analysis, cells were stained with propidium iodide and annexin $\mathrm{V}$ and analyzed by FACS.

\section{Real-time qPCR}

After removal of genomic DNA by treatment with DNase (Wako Nippon Gene), randomly primed cDNA strands were generated with reverse transcriptase III (Invitrogen). RNA expression was quantified by real-time PCR with gene-specific primers, and the values were normalized to the expression of Rps18 mRNA. qPCR was performed with the Fast SYBR Green Master Mix (Applied Biosystems). Data were collected and calculated by using the StepOnePlus real-time PCR system (Applied Biosystems).

\section{Reagents and Abs}

The STING ligands CGAMP and c-di-AMP were purchased from Invivogen. DMXAA and etoposide were obtained from Sigma-Aldrich. Z-VAD-FMK and Rapamycin were obtained from Calbiochem.

Abs specific for anti-cyclin A (C-19, 1:1,000 dilution), anti-cyclin B1 ( $H-433,1: 1,000$ dilution), anti-cyclin $E$ (M-20, 1:1,000 dilution), antiCdk1 (17, 1:1,000 dilution), and anti-Cdk2 (H298, 1:1,000 dilution); antiphospho-S6K1 (\#9205, 1:1,000 dilution), anti-phospho-S6 (\#2211, 1:1,000 dilution), anti-phospho-4E-BP1 (\#9459, 1:1,000), anti-phosphoAkt (\#9271, 1:1,000 dilution), anti-phospho-IRF3 (\#4947, 1:1,000 dilution), anti-phospho-STAT5 (\#9351, 1:1,000 dilution), anti-phospho JAK3 (\#5031, 1:1,000 dilution), anti-STING (\#13647, 1:1,000 dilution), anti-phospho-TBK1 (\#5483, 1:1,000 dilution), anti-ERK (\#9102, 1: 1,000 dilution), anti-cleaved PARP (\#9548, 1:1,000 dilution), anti-cleaved Caspase-3 (\#9661, 1:1,000 dilution), anti-TBK1 (\#3013, 1:1,000 dilution), anti-IKKE (\#2690, 1:1,000 dilution), and anti-IRF3 (\#4302, 1:1,000 dilution) were obtained from Cell signaling Technology; anti-IRF7 (EPR4718, 1:1,000 dilution) was obtained from Abcam; anti-CD98 FITC (10.3, 1:50 dilution) was obtained from MBL. Flow cytometric analysis was performed on a FACSCalibur or LSR Fortessa X-20 and data were analyzed with CellQuest Pro or FlowJo.

\section{Western blot analysis}

Cells were lysed in 1\% Nonidet P-40 (NP-40) lysis buffer (1\% NP-40, $50 \mathrm{mM}$ Tris, $150 \mathrm{mM} \mathrm{NaCl}, 5 \mathrm{mM}$ EDTA, $10 \mu \mathrm{g} / \mathrm{ml}$ of aprotinin, $12.5 \mu \mathrm{g} / \mathrm{ml}$ of chymostatin, $50 \mu \mathrm{g} / \mathrm{ml}$ of leupeptin, $25 \mu \mathrm{g} / \mathrm{ml}$ of 
pepstatin A, $1 \mathrm{mM}$ phenylmethylsulfonyl fluoride, and $2 \mathrm{mM} \mathrm{Na}_{3} \mathrm{VO}_{4}$ ). The lysates or immunoprecipitates were subjected by SDS-PAGE, and Western blots were carried out for the transferred membrane by reacting with specific $A b$ and developed with an enhanced chemiluminescence assay according to the manufacturer's recommendations (Pierce).

\section{Retroviral transduction}

4E-BP1 (T37/46 AA) was cloned into the retroviral vector pMIG (provided by T. Kitamura, University of Tokyo). The construct was transiently transduced into Phoenix packaging cells (provided by $G$ Nolan, Stanford University) using Lipofectamine with PLUS reagent (Invitrogen). Naive $\mathrm{CD} 4^{+} \mathrm{T}$ cells were stimulated with plate-bound anti-CD3/CD28 Abs, and the cells were transduced by centrifugation at $1,640 \mathrm{~g}$ for $120 \mathrm{~min}$ in retroviral supernatants plus $8 \mu \mathrm{g} / \mathrm{ml}$ of polybrene (Sigma-Aldrich) on day 1 after stimulation. After $72 \mathrm{~h}$ of stimulation, the cells were sorted with a FACSAria to obtain GFPpositive populations.

\section{RNA-seq analysis}

Total RNA was isolated from T cells using Direct-zol RNA kits (ZYMO RESEARCH) according to the manufacturer's instructions. The DNA library for RNA-seq analysis was constructed with NEBNext Ultra RNA Library Prep Kit for Illumina (NEB Biolabs, Inc) according to the manufacturer's instruction. The size range of the resulting DNA library was estimated on a 2100 Bioanalyzer (Agilent Technologies). The DNA library was subjected to the HiSeq 1500 sequencing system (Illumina) in a single-end read mode to obtain the sequencing data. The sequence reads were mapped to the Mus musculus reference genome (NCBI version 37) using TopHat2 version 2.0.8 and botwie2 version 2.1.0 with default parameters, and gene annotation was provided by NCBI. According to the mapped data, Cufflinks (version 2.1.1) was used to calculate the FPKM (fragments per kilobase per million mapped reads) values. Pathway-enrichment analysis was performed using DAVID Bioinformatics Resources 6.8 (Huang da et al, 2009). Heat maps were produced from normalized expression data referring to DAVID Bioinformatics Resources 6.8.

\section{Lipid analysis}

Lipid extraction of $\mathrm{CD}^{+} \mathrm{T}$ cells was performed as described (Tsugawa et al, 2017). Briefly, chloroform $(100 \mu \mathrm{l}$ ) was added to dried cells in a tube, followed by 30 s sonication. After 60 -min incubation at room temperature, $200 \mu \mathrm{l}$ of $\mathrm{MeOH}$ was added and vortexed for $10 \mathrm{~s}$. After $120 \mathrm{~min}$ of incubation, $20 \mu \mathrm{l}$ of Milli-Q water was added, vortexed again, and the tube was left to stand for $10 \mathrm{~min}$. The tubes were then centrifuged at 2,000 $\mathrm{g}$ for $10 \mathrm{~min}$ at $20^{\circ} \mathrm{C}$, and the supernatant was transferred to LC-MS vials.

LC-MS/MS and LC-MS were used for identification and quantification of lipids. Non-targeted lipidomics analysis was performed as described (Takatani et al, 2015; Hirabayashi et al, 2017). Briefly, dried total lipid extracts were redissolved in $50 \mathrm{ml}$ of chloroform: methanol (2:1, vol/vol), and $2 \mathrm{ml}$ of samples were separated by an ACQUITY UPLC BEH C18 column (50_2.1 mm i.d., particle size $1.7 \mu \mathrm{m}$, Waters) at a flow rate of $300 \mu \mathrm{l} / \mathrm{min}$ at $45^{\circ} \mathrm{C}$ using an ACQUITY UPLC system (Waters) equipped with a binary pump and automatic sample injector. Solvent A consisted of acetonitrile/methanol/ water (20:20:60, vol/vol/vol) and solvent B was isopropanol, both containing $5 \mathrm{mM}$ ammonium acetate. The solvent composition started at 100\% A for the first $1 \mathrm{~min}$ and was changed linearly to $64 \%$ $B$ at $7.5 \mathrm{~min}$, where it was held for $4.5 \mathrm{~min}$. The gradient was increased linearly to $82.5 \%$ B at $12.5 \mathrm{~min}$, followed by $85 \%$ B at 19 min and $95 \%$ B at 20 min before re-equilibrating the column with $100 \% \mathrm{~A}$ for 5 min. Qualitative and quantitative analysis of lipids was performed by MS and data-dependent MS/MS acquisition with a scan range of $m / z 70-1,250$ using a Triple TOF $5600^{+}$System (AB SCIEX) in the negative and positive ion mode. Raw data files from the TOF-MS were converted to MGF files using the program AB SCIEX MS converter for subsequent quantitative analysis with 2DICAL (Mitsui Knowledge Industry). Identification of molecular species was accomplished by comparison with retention times and MS/MS spectra with commercially available standards or reference samples.

\section{In vivo tumor growth and treatment}

B16 F10 melanoma cells were cultured in complete DMEM media supplemented $10 \%$ heat-inactivated FBS. RAG1-KO mice were reconstituted with $2 \times 10^{6}$ Sting $^{+/-}$or Sting ${ }^{-/-}$T cells and $4 \times 10^{6}$ WT $\mathrm{B}$ cells. After more than $30 \mathrm{~d}$ after reconstitution, $\mathrm{T}$ cell-specific STING-KO mice and control mice were injected s.c. on the back with a total of $2 \times 10^{5}$ B16 F10 cells on day 0 . On days 8,10 , and 13 , the mice were given intratumor injections of cGAMP $(10 \mu \mathrm{g})$, and the mice were monitored for tumor growth and mortality. The tumor area was measured with a digital caliper and calculated using the formula: largest diameter $\times$ smallest diameter.

\section{Statistics}

Statistical significance was determined by a two-tailed unpaired $t$ test. $P<0.05$ was considered statistically significant.

\section{Data Availability}

RNA sequencing data can be found publicly available on the National Center for Biotechnology Information (NCBI) Gene Expression Omnibus (GEO) website under the accession number for GSE104725.

\section{Supplementary Information}

Supplementary Information is available at https://doi.org/10.26508/lsa. 201800282

\section{Acknowledgements}

We thank T Taniguchi for providing IRF3-KO, IRF7-KO, and IRF3/7-DKO mice; T Yokosuka, A Takeuchi, S Tsukumo, R Onishi, A Hashimoto-Tane, M Badr, N Hayatsu, H Ishigame, $\mathrm{H}$ Negishi, K Miyauchi, M Kubo, and H Hara for discussions and experimental help; M Sakuma for technical support; P Burrows for helpful comments on the manuscript; and M Yoshioka for secretarial 
assistance. This work was supported by a Grant-in-Aid for Scientific Research from the Japan Society for the Promotion of Science KAKENHI (grants 16K08852 for T Imanishi and 24229004 for T Saito).

\section{Author Contributions}

T Imanishi: conceptualization, data curation, formal analysis, funding acquisition, validation, investigation, and writing-original draft and project administration.

M Unno: investigation and data curation.

W Kobayashi: investigation and data curation.

N Yoneda: investigation and data curation.

$S$ Matsuda: investigation and resources.

K Ikeda: investigation and data curation.

T Hoshii: resources.

A Hirao: resources.

K Miyake: resources.

GN Barber: resources.

M Arita: investigation and data curation.

KJ Ishii: resources.

S Akira: resources.

T Saito: conceptualization, supervision, funding acquisition, validation, project administration, and writing-review and editing.

\section{Conflict of Interest Statement}

The authors declare that they have no conflict of interest.

\section{References}

Burdette DL, Monroe KM, Sotelo-Troha K, Iwig JS, Eckert B, Hyodo M, Hayakawa $Y$, Vance RE (2011) STING is a direct innate immune sensor of cyclic diGMP. Nature 478: 515-518. doi:10.1038/nature10429

Cavlar T, Deimling T, Ablasser A, Hopfner KP, Hornung V (2013) Speciesspecific detection of the antiviral small-molecule compound CMA by STING. EMBO / 32: 1440-1450. doi:10.1038/emboj.2013.86

Cerboni S, Jeremiah N, Gentili M, Gehrmann U, Conrad C, Stolzenberg MC, Picard C, Neven B, Fischer A, Amigorena S, et al (2017) Intrinsic antiproliferative activity of the innate sensor STING in T lymphocytes. J Exp Med 214: 1769-1785. doi:10.1084/jem.20161674

Chattopadhyay S, Kuzmanovic T, Zhang Y, Wetzel JL, Sen GC (2016) Ubiquitination of the transcription factor IRF-3 activates RIPA, the apoptotic pathway that protects mice from viral pathogenesis. Immunity 44: 1151-1161. doi:10.1016/j.immuni.2016.04.009

Chattopadhyay S, Marques JT, Yamashita M, Peters KL, Smith K, Desai A, Williams BR, Sen GC (2010) Viral apoptosis is induced by IRF-3mediated activation of Bax. EMBO / 29: 1762-1773. doi:10.1038/ emboj.2010.50

Chen L, Flies DB (2013) Molecular mechanisms of T cell co-stimulation and co-inhibition. Nat Rev Immunol 13: 227-242. doi:10.1038/nri3405

Chi H (2012) Regulation and function of mTOR signalling in T cell fate decisions. Nat Rev Immunol 12: 325-338. doi:10.1038/nri3198

Colina R, Costa-Mattioli M, Dowling RJ, Jaramillo M, Tai LH, Breitbach CJ, Martineau Y, Larsson O, Rong L, Svitkin YV, et al (2008) Translational control of the innate immune response through IRF-7. Nature 452: 323-328. doi:10.1038/nature06730

Cottalorda A, Verschelde C, Marcais A, Tomkowiak M, Musette P, Uematsu S, Akira S, Marvel J, Bonnefoy-Berard N (2006) TLR2 engagement on CD8
T cells lowers the threshold for optimal antigen-induced T cell activation. Eur J Immunol 36: 1684-1693. doi:10.1002/eji.200636181

Demaria O, De Gassart A, Coso S, Gestermann N, Di Domizio J, Flatz L, Gaide O, Michielin O, Hwu P, Petrova TV, et al (2015) STING activation of tumor endothelial cells initiates spontaneous and therapeutic antitumor immunity. Proc Natl Acad Sci USA 112: 15408-15413. doi:10.1073/ pnas.1512832112

Deng L, Liang H, Xu M, Yang X, Burnette B, Arina A, Li XD, Mauceri H, Beckett M, Darga T, et al (2014) STING-dependent cytosolic DNA sensing promotes radiation-induced type I interferon-dependent antitumor immunity in immunogenic tumors. Immunity 41: 843-852. doi:10.1016/ j.immuni.2014.10.019

Diner EJ, Burdette DL, Wilson SC, Monroe KM, Kellenberger CA, Hyodo M, Hayakawa Y, Hammond MC, Vance RE (2013) The innate immune DNA sensor cGAS produces a noncanonical cyclic dinucleotide that activates human STING. Cell Rep 3: 1355-1361. doi:10.1016/j.celrep. 2013.05.009

Duran RV, Oppliger W, Robitaille AM, Heiserich L, Skendaj R, Gottlieb E, Hall MN (2012) Glutaminolysis activates Rag-mTORC1 signaling. Mol Cell 47: 349-358. doi:10.1016/j.molcel.2012.05.043

Eaton JM, Mullins GR, Brindley DN, Harris TE (2013) Phosphorylation of lipin 1 and charge on the phosphatidic acid head group control its phosphatidic acid phosphatase activity and membrane association. J Biol Chem 288: 9933-9945. doi:10.1074/jbc.m112.441493

Gao D, Li T, Li XD, Chen X, Li QZ, Wight-Carter M, Chen Z) (2015) Activation of cyclic GMP-AMP synthase by self-DNA causes autoimmune diseases. Proc Natl Acad Sci USA 112: E5699-E5705. doi:10.1073/pnas.1516465112

Gao P, Ascano M, Zillinger T, Wang W, Dai P, Serganov AA, Gaffney BL, Shuman S, Jones RA, Deng L, et al (2013) Structure-function analysis of STING activation by $c\left[\mathrm{G}\left(2^{\prime}, 5^{\prime}\right) \mathrm{pA}\left(3^{\prime}, 5^{\prime}\right) \mathrm{p}\right]$ and targeting by antiviral DMXAA. Cell 154: 748-762. doi:10.1016/j.cell.2013.07.023

Gulen MF, Koch U, Haag SM, Schuler F, Apetoh L, Villunger A, Radtke F, Ablasser A (2017) Signalling strength determines proapoptotic functions of STING. Nat Commun 8: 427. doi:10.1038/s41467-01700573-w

Han GS, Wu WI, Carman GM (2006) The Saccharomyces cerevisiae Lipin homolog is a Mg2+-dependent phosphatidate phosphatase enzyme. J Biol Chem 281: 9210-9218. doi:10.1074/jbc.m600425200

Hirabayashi T, Anjo T, Kaneko A, Senoo Y, Shibata A, Takama H, Yokoyama K, Nishito Y, Ono T, Taya C, et al (2017) PNPLA1 has a crucial role in skin barrier function by directing acylceramide biosynthesis. Nat Commun 8: 14609. doi:10.1038/ncomms14609

Huang da W, Sherman BT, Lempicki RA (2009) Systematic and integrative analysis of large gene lists using DAVID bioinformatics resources. Nat Protoc 4: 44-57. doi:10.1038/nprot.2008.211

Imanishi T, Hara H, Suzuki S, Suzuki N, Akira S, Saito T (2007) Cutting edge: TLR2 directly triggers Th1 effector functions. J Immunol 178: 6715-6719. doi:10.4049/jimmunol.178.11.6715

Imanishi T, Ishihara C, Badr Mel S, Hashimoto-Tane A, Kimura Y, Kawai T, Takeuchi O, Ishii KJ, Taniguchi S, Noda T, et al (2014) Nucleic acid sensing by T cells initiates Th2 cell differentiation. Nat Commun 5: 3566. doi:10.1038/ncomms4566

Ishii KJ, Coban C, Kato H, Takahashi K, Torii Y, Takeshita F, Ludwig H, Sutter G, Suzuki K, Hemmi H, et al (2006) A Toll-like receptor-independent antiviral response induced by double-stranded B-form DNA. Nat Immunol 7: 40-48. doi:10.1038/ni1282

Ishikawa H, Barber GN (2008) STING is an endoplasmic reticulum adaptor that facilitates innate immune signalling. Nature 455: 674-678. doi:10.1038/nature07317

Ishikawa H, Ma Z, Barber GN (2009) STING regulates intracellular DNAmediated, type I interferon-dependent innate immunity. Nature 461 788-792. doi:10.1038/nature08476 
Kidani Y, Elsaesser H, Hock MB, Vergnes L, Williams KJ, Argus JP, Marbois BN, Komisopoulou E, Wilson EB, Osborne TF, et al (2013) Sterol regulatory element-binding proteins are essential for the metabolic programming of effector T cells and adaptive immunity. Nat Immunol 14: 489-499. doi:10.1038/ni.2570

Komai-Koma M, Jones L, Ogg GS, XU D, Liew FY (2004) TLR2 is expressed on activated T cells as a costimulatory receptor. Proc Natl Acad Sci USA 101: 3029-3034. doi:10.1073/pnas.0400171101

Larkin B, Ilyukha V, Sorokin M, Buzdin A, Vannier E, Poltorak A (2017) Cutting edge: Activation of STING in T cells induces type I IFN responses and cell death. J Immunol 199: 397-402. doi:10.4049/jimmunol.1601999

Medzhitov R (2001) Toll-like receptors and innate immunity. Nat Rev Immunol 1: 135-145. doi:10.1038/35100529

Moretti J, Roy S, Bozec D, Martinez J, Chapman JR, Ueberheide B, Lamming DW, Chen Z), Horng T, Yeretssian G, et al (2017) STING senses microbial viability to orchestrate stress-mediated autophagy of the endoplasmic reticulum. Cell 171: 809-823 e813. doi:10.1016/j. cell.2017.09.034

Pearce LR, Alton GR, Richter DT, Kath JC, Lingardo L, Chapman J, Hwang C, Alessi DR (2010) Characterization of PF-4708671, a novel and highly specific inhibitor of p70 ribosomal S6 kinase (S6K1). Biochem J 431: 245-255. doi:10.1042/bj20101024

Reynolds JM, Pappu BP, Peng J, Martinez GJ, Zhang Y, Chung Y, Ma L, Yang XO, Nurieva RI, Tian Q, et al (2010) Toll-like receptor 2 signaling in CD4(+) T lymphocytes promotes $T$ helper 17 responses and regulates the pathogenesis of autoimmune disease. Immunity 32: 692-702. doi:10.1016/j.immuni.2010.04.010

Roth S, Rottach A, Lotz-Havla AS, Laux V, Muschaweckh A, Gersting SW, Muntau AC, Hopfner KP, Jin L, Vanness K, et al (2014) Rad50-CARD9 interactions link cytosolic DNA sensing to IL-1beta production. Nat Immunol 15: 538-545. doi:10.1038/ni.2888

Sanchez EL, Lagunoff M (2015) Viral activation of cellular metabolism. Virology 479-480: 609-618. doi:10.1016/j.virol.2015.02.038

Sun L, Wu J, Du F, Chen X, Chen ZJ (2013) Cyclic GMP-AMP synthase is a cytosolic DNA sensor that activates the type I interferon pathway. Science 339: 786-791. doi:10.1126/science.1232458

Takaoka A, Wang Z, Choi MK, Yanai H, Negishi H, Ban T, Lu Y, Miyagishi M, Kodama T, Honda K, et al (2007) DAI (DLM-1/ZBP1) is a cytosolic DNA sensor and an activator of innate immune response. Nature 448: 501-505. doi:10.1038/nature06013

Takatani N, Use K, Kato A, Ikeda K, Kojima K, Aichi M, Maeda S, Omata T (2015) Essential role of acyl-ACP synthetase in acclimation of the cyanobacterium synechococcus elongatus strain PCC 7942 to highlight conditions. Plant Cell Physiol 56: 1608-1615. doi:10.1093/pcp/ pcr086

Takeda K, Kaisho T, Akira S (2003) Toll-like receptors. Annu Rev Immunol 21 335-376. doi:10.1146/annurev.immunol.21.120601.141126

Temizoz B, Kuroda E, Ohata K, Jounai N, Ozasa K, Kobiyama K, Aoshi T, Ishii KJ (2015) TLR9 and STING agonists synergistically induce innate and adaptive type-II IFN. Eur J Immunol 45: 1159-1169. doi:10.1002/eji. 201445132

Tsugawa H, Ikeda K, Tanaka W, Senoo Y, Arita M, Arita M (2017) Comprehensive identification of sphingolipid species by in silico retention time and tandem mass spectral library. J Cheminformatics 9: 19. doi:10.1186/ s13321-017-0205-3

Wang H, Hu S, Chen X, Shi H, Chen C, Sun L, Chen ZJ (2017) CGAS is essential for the antitumor effect of immune checkpoint blockade. Proc Natl Acad Sci USA 114: 1637-1642. doi:10.1073/pnas.1621363114

Woo SR, Fuertes MB, Corrales L, Spranger S, Furdyna MJ, Leung MY, Duggan R, Wang Y, Barber GN, Fitzgerald KA, et al (2014) STING-dependent cytosolic DNA sensing mediates innate immune recognition of immunogenic tumors. Immunity 41: 830-842. doi:10.1016/j. immuni.2014.10.017

Wu J, Sun L, Chen X, Du F, Shi H, Chen C, Chen ZJ (2013) Cyclic GMP-AMP is an endogenous second messenger in innate immune signaling by cytosolic DNA. Science 339: 826-830. doi:10.1126/science.1229963

Yamasaki S, Ishikawa E, Kohno M, Saito T (2004) The quantity and duration of FcRgamma signals determine mast cell degranulation and survival. Blood 103: 3093-3101. doi:10.1182/blood-2003-08-2944

Yang K, Shrestha S, Zeng H, Karmaus PW, Neale G, Vogel P, Guertin DA, Lamb RF, Chi H (2013) T cell exit from quiescence and differentiation into Th2 cells depend on Raptor-mTORC1-mediated metabolic reprogramming. Immunity 39: 1043-1056. doi:10.1016/j.immuni. 2013.09.015

Zhao Z, Condomines M, van der Stegen SJC, Perna F, Kloss CC, Gunset G, Plotkin J, Sadelain M (2015) Structural design of engineered costimulation determines tumor rejection kinetics and persistence of CAR T cells. Cancer Cell 28: 415-428. doi:10.1016/j.ccell.2015.09.004

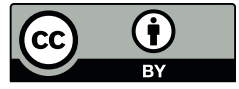

License: This article is available under a Creative Commons License (Attribution 4.0 International, as described at https://creativecommons.org/ licenses/by/4.0/). 